\title{
Functionally Substituted Arylcopper Compounds: Synthesis, Structure, and Reactivity of (2-(2-Oxazolinyl)aryl)copper(I) Species
}

\author{
Erik Wehman, ${ }^{1 \mathrm{a}}$ Gerard van Koten, ${ }^{*}, 1 \mathrm{a}$ Johann T. B. H. Jastrzebski, ${ }^{\text {1a }}$ Marc A. Rotteveel, ${ }^{\text {tb }}$ and \\ Casper H. Stam ${ }^{16}$ \\ Anorganisch Chemisch Laboratorium and Laboratorium voor Kristallografle, J. $H$. van't Hoff Instituut, University \\ of Amsterdam, Neuwe Achtergracht 166, 1018 WV Amsterdam, The Netherlands
}

Received July 8, 1987

\begin{abstract}
Arylcopper(I) compounds containing an oxazoline substituent in the position ortho to the $\mathrm{Cu}-\mathrm{C}$ bond have been synthesized from the corresponding aryllithium compounds and $\mathrm{CuBr}$. Depending on the order of addition this reaction afforded either a pure arylcopper(I) compound (slow addition of $\mathrm{CuBr}$ to the aryllithium reagent) or a mixture of arylcopper(I) and its $\mathrm{CuBr}$ adduct with $\left[(\mathrm{RCu})_{2} \mathrm{CuBr}\right]_{2}$ stoichiometry (addition of aryllithium to a $\mathrm{CuBr}$ suspension). The new arylcopper(I) compounds were characterized by ${ }^{1} \mathrm{H}$ NMR spectroscopy and cryoscopic molecular weight determinations in benzene, and for two of them the X-ray crystal structure has been determined. The structure of $[\mathrm{Cu}(\mathrm{MeOXL})]_{2}(\mathrm{MeOXL}=2-(4,4-$ dimethyl-2-oxazolinyl)-5-methylphenyl) (2b) (monoclinic crystals of space group $P 2 / n, Z=8$, with $a=$ 19.050 (1) $\AA, b=6.671$ (1) $\AA, c=18.927$ (1) $\AA$, and $\left.\beta=106.49(1)^{\circ}\right)$ shows a dimeric molecule containing a bridging 2-(2-oxazolinyl)aryl moiety, with two-center, two-electron carbon-copper bonds $(\mathrm{Cu}-\mathrm{C}=1.899$ (5) $\AA$ ). The oxazoline substituent is coordinated via the nitrogen with a $\mathrm{Cu}-\mathrm{N}$ distance of 1.902 (4) $\AA$. Such a two-center, two-electron bridge bonding mode is unprecedented in arylcopper chemistry. $\mathrm{The} \mathrm{CuBr}$ adduct of $\mathbf{2 b}\left[\mathrm{Cu}_{2}(\mathrm{MeOXL})_{2} \mathrm{CuBr}\right]_{2}$ (triclinic crystals of space group $P \overline{1}, Z=2$, with $a=13.661$ (3) $\AA, b$ $=19.154(4) \AA, c=11.666(5) \AA, \alpha=95.46(3)^{\circ}, \beta=92.49(3)^{\circ}$, and $\left.\gamma=110.49(3)^{\circ}\right)$ also shows a dimeric structure containing six copper atoms which form a distorted octahedron with four three-center, two-electron bridging aryl groups $(\mathrm{Cu}-\mathrm{C}=2.031$ (3) $\AA$ (mean); $\mathrm{Cu}-\mathrm{Cu}=2.436$ (3) $\AA$ (mean)) and two three-center, four-electron bridging bromine atoms ( $\mathrm{Cu}-\mathrm{Br}=2.524$ (3) $\AA$ (mean); $\mathrm{Cu}-\mathrm{Cu}=3.023$ (4) $\AA$ (mean)). The oxazoline substituent is coordinated via the nitrogen atom $(\mathrm{Cu}-\mathrm{N}=1.968(4) \AA$ (mean)). Ullmann biaryl coupling reactions of the new organocopper(I) compounds with 2-iodopiperonal imine were little influenced by a hetero substituent in either the arylcopper(I) compound or the aryl iodide anchoring group. Moreover, since the addition of $\mathrm{P}(\mathrm{OMe})_{3}$ did not influence the coupling reaction, it can be concluded that "external" ligands do not have a critical role in these reactions. The results of this study show clearly the important influence that an ortho-chelating substituent (i.e. the 2-oxazolinyl group in the present compounds) can have on both the structural features and the reactivity of the arylcopper compounds.
\end{abstract}

\section{Introduction}

Among the organometallic reagents used in organic synthesis, organocopper compounds are probably some of the most widely used since the great diversity of accessible organocopper compounds, each with its specific reactivity, makes them very attractive reagents. ${ }^{2}$

A particularly useful copper-mediated reaction is the Ullmann biaryl coupling of aryl iodides ${ }^{3}$ which, in spite of the shortcomings associated with the use of copper bronze in this reaction, has proven its value for decades. The use of copper bronze often leads in this reaction to a mixture of symmetric and asymmetric coupled products, but this problem can be overcome now that it is recognized that arylcopper species are the reactive intermediates. ${ }^{4}$ This knowledge has been nicely used by Ziegler et al. in their synthesis of $( \pm)$-Steganacin, an antileukemic lactone containing a biphenyl moiety, ${ }^{5}$ that employs intramolecularly coordinated arylcopper reagents. Their studies showed that the use of the latter compounds gave a very selective cross-coupling reaction with aryl iodides at ambient temperature. Interesting differences were found in the reactivity of various aryl iodides as well as of the organocopper intermediates. Whereas aryl iodides containing a nitrogen or sulfur-containing ortho substituent reacted with organocopper compounds very selectively to afford cross-coupled products, those containing an ester function

*To whom correspondence should be addressed at the Laboratory of Organic Chemistry, Department of Metal Mediated Synthesis, University of Utrecht, Padualaan 8, $3584 \mathrm{CH}$ Utrecht, The Netherlands. reacted to afford a mixture of symmetrical and unsymmetrical coupled products, as well as products orginating from halogen exchange and reduced products. The coupling reactions in which only one of the reactants bore a coordinating substituent afforded low yields of cross-coupled products. It was assumed that coordination of the aryl iodide to a copper center of an organocopper intermediate preceded the actual coupling reaction though attempts to isolate organocopper species occurring in this reaction sequence failed. ${ }^{6}$

It is now well-known that many organocopper compounds are polynuclear. ${ }^{7}$ However, the above-mentioned

(1) (a) Anorganisch Chemisch Laboratorium. (b) Laboratorium voor Kristallografie.

(2) (a) Carruthers, W. In "Comprehensive Organometallic Chemistry; Wilkinson, G., Stone, F. G. A., Abel, E. W., Eds.; Pergamon: Oxford, 1982; Vol. 7, Chapter 49. (b) Posner, G. H. An Introduction to Synthesis using Organocopper Reagents; Wiley-Interscience: New York, 1980.

(3) (a) Fanta, P. E. Synthesis 1974, 9. (b) Goshaev, M.; Otroshchenko, O. S.; Sadykov, A. S. Russ. Chem. Rev. (Engl. Transl.) 1972, 41, 1046.

(4) (a) Lewin, A. H.; Cohen, T. Tetrahedron Lett. 1965, 4531. (b) Nilsson, M.; Ullenius, C. Acta Chem. Scand. 1970, 24, 2379. (c) Nilsson, M.; Wennerström, O. Acta Chem. Scand. 1970, 24, 482. (d) Nilsson, M.; Wennerström, O. Tetrahedron Lett. 1968, 3307. (e) van Koten, G.; ten Hoedt R. W. M.; Noltes, J. G. J. Org. Chem. 1977, 42, 2705.

(5) (a) Ziegler, F. E.; Fowler, K. W.; Kanfer, S. J. Am. Chem. Soc. 1976 98, 8282. (b) Ziegler, F. E.; Fowler, K. W.; Sinha, N. D. Tetrahedron Lett. 1978, 2767. (c) Ziegler, F. E.; Chliwner, I.; Fowler, K. W.; Kanfer, S. J.; Kuo, S. J.; Sinha, N. D. J. Am. Chem. Soc, 1980, $102,790$.

(6) Ziegler, F. E., personal communication.

(7) (a) Camus, A.; Marsich, N.; Nardin, G.; Randaccio, L. Inorg. Chim. Acta 1977, 23, 131. (b) van Koten, G.; Noltes, J. G. In Comprehensive Organometallic Chemistry; Wilkinson, G., Stone, F. G. A., Abel, E. W. Eds.; Pergamon: Oxford, 1982; Vol. 1, Chapter 14. 
cross-coupling reactions seem to involve mononuclear copper intermediates. This seemed surprising in view of our earlier observation that organocopper compounds with ortho-chelating aryl groups are aggregated species $\mathrm{Cu}_{n} \mathrm{Ar}_{n}$ (e.g. $\left.\mathrm{Cu}_{4}\left(\mathrm{C}_{6} \mathrm{H}_{4} \mathrm{CH}_{2} \mathrm{NMe}_{2}-2-\mathrm{Me}-5\right)_{4}\right)$. These aggregates, moreover, are capable of forming mixed arylcopper halide species $\mathrm{Cu}_{n+m} \mathrm{Ar}_{n} \mathrm{X}_{m}$ (e.g. $\left.\mathrm{Cu}_{6}\left(\mathrm{C}_{6} \mathrm{H}_{4} \mathrm{NMe}_{2}-2\right){ }_{4} \mathrm{Br}_{2}\right)$ by reaction with copper(I) halides, $\mathrm{CuX}$, or organocopper species, $\mathrm{CuR}$ (e.g. $\left.\mathrm{Cu}_{6}\left(\mathrm{C}_{6} \mathrm{H}_{4} \mathrm{NMe}_{2}-2\right)_{4}\left(\mathrm{C} \equiv \mathrm{CC}_{6} \mathrm{H}_{4} \mathrm{CH}_{3}-4\right)_{2}\right)$. It was found that the nature of the ortho substituent exerts a strong influence on the type of aggregate formed. This result can be explained as resulting from the constraints involved in formation of a chelate ring by coordination of the ortho substituent heteroatom to copper. It was therefore of interest to us to embark on the study of (2(2-oxazolinyl)aryl)copper(I) compounds. The objectives of the study presented in this paper were (1) the synthesis and structural characterization of the organocopper compounds, (2) the determination of the influence of the heteroatom substituent in the aryl groups on these coupling reactions, and (3) an examination of the influence of coligands on the reactions. A part of this work has been reported as a preliminary communication. ${ }^{8}$

\section{Results and Discussion}

Synthesis of the Compounds. A convenient route for the synthesis of pure organocopper compounds is the reaction of an organolithium compound with $\mathrm{CuBr} .^{9}$ Lithiated aryloxazolines can be prepared easily by direct lowtemperature metalation of the corresponding hydrocarbon with $n$-BuLi in THF. ${ }^{10}$ However, reaction of $\mathrm{Li}$ (OXL) (OXL = 2-(4,4-dimethyl-2-oxazolinyl)phenyl), ${ }^{11}$ obtained via direct metalation of $\mathrm{OXL}-\mathrm{H}$ in $\mathrm{THF}$, with $\mathrm{CuBr}$ failed to give the desired organocopper compound [ $\mathrm{Cu}(\mathrm{OXL})]$ in a pure form. Although the reason for this failure has not been studied further, it is likely that products originating from an addition reaction of $n$-BuLi to the imine bond of the oxazoline group ${ }^{12}$ hamper the isolation of the pure arylcopper compound.

Pure Li(OXL) (1a) and Li(MeOXL) (1b) can be prepared by direct metalation of OXL-H and MeOXL-H, respectively, with $n-\mathrm{BuLi}$ in hexane. This reaction affords a white precipitate of these aryllithium compounds whereas eventual side products remain in solution and can be removed by filtration. Proof for the site of lithiation was obtained from deuteriolysis experiments of $\mathbf{1 a}$ and $\mathbf{1 b}$ with $\mathrm{D}_{2} \mathrm{O}$ and from the reaction of $1 \mathbf{b}$ with $\mathrm{Me}_{3} \mathrm{SiCl}$, affording the silylated oxazoline 6 . These reactions are summarized in eq 1.

The lithium compounds $\mathbf{1 a}$ and $\mathbf{1 b}$, which are obtained as white solids in $70-80 \%$ yield, show a pyrophoric reaction upon exposure to air. Since they can be stored at room temperature under an atmosphere of nitrogen for only a

(8) Wehman, E.; van Koten, G.; Jastrzebski, J. T. B. H. J. Organomet. Chem. 1986, 302, C35.

(9) (a) Costa, G.; Camus, A.; Gatti, L.; Marsich, N. J. Organomet. Chem. 1966, 5, 568. (b) van Koten, G.; Leusink, A. J.; Noltes, J. G. J. Chem. Soc. Chem. Commun 1970, 1107. (c) van Koten, G.; Leusink, A J.; Noltes, J. G. Inorg. Nucl. Chem. Lett. 1971, 227.

(10) (a) Gschwend, G. W.; Hamdan, A. J. Org. Chem. 1975, 40, 2008. (b) Meyers, A. I.; Mihelich, E. D. J. Org. Chem. 1975, 40, 3158.

(11) Throughout this paper 2-(4,4-dimethyl-2-oxazolinyl)phenyl will be abbreviated as OXL and the p-methyl derivative as MeOXL. The hydrocarbons are represented as OXL-H and MeOXL-H, respectively, whereas the metal complexes are represented as $M(O X L)$ and $M$ (MeOXL), respectively.

(12) It has been reported that addition to the imine bond by $n$-BuLi can occur at temperatures above $-20^{\circ} \mathrm{C} .^{13}$ 1372 .

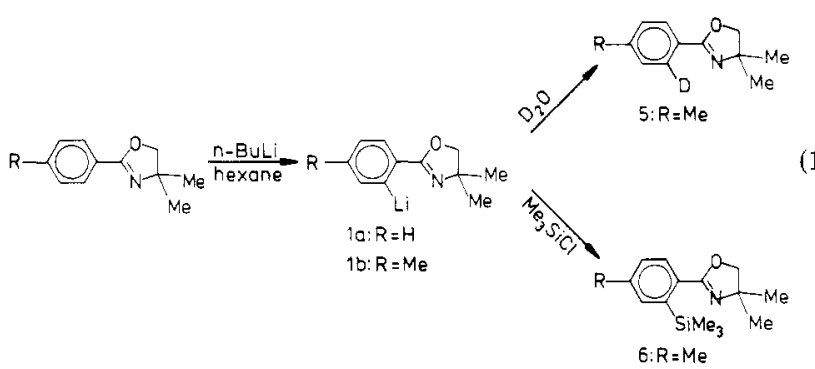

few hours, the best results for subsequent reactions with $\mathrm{CuBr}$ are obtained with freshly prepared samples.

The 1:1 molar reaction of $\mathrm{Li}(\mathrm{OXL})$ and $\mathrm{CuBr}$ in diethyl ether resulted in an orange compound, $2 \mathrm{a}$, which analyzed as $\mathrm{Cu}(\mathrm{OXL})$. Reaction of $\mathrm{Li}(\mathrm{MeOXL})$ with $\mathrm{CuBr}$ in diethyl ether gave different products depending on the order of addition of the reactants. Addition of a solution of $\mathrm{Li}(\mathrm{MeOXL})$ in diethyl ether to a suspension of $\mathrm{CuBr}$ in the same solvent (1:1 $\mathrm{Li}(\mathrm{MeOXL}) / \mathrm{CuBr}$ ratio) afforded a bright orange product which was indicated by its elemental analyses to be a mixture of the organocopper compound [ $\mathrm{Cu}(\mathrm{MeOXL})](2 b)$ and its $\mathrm{CuBr}$ complex $\left[\mathrm{Cu}_{2}(\mathrm{MeOXL})_{2} \mathrm{CuBr}\right]_{2}(3)$. However, reversed addition, i.e. slow addition of solid $\mathrm{CuBr}$ to a solution of $\mathrm{Li}(\mathrm{MeOXL})$ in diethyl ether, gave pure $[\mathrm{Cu}(\mathrm{MeOXI})](2 \mathrm{~b})$. Reaction of [Cu(MeOXL)] with $\mathrm{CuBr}$ in a 2:1 molar ratio afforded the organocopper-copper bromide adduct complex 3 which cannot be transformed to pure $[\mathrm{Cu}(\mathrm{MeOXL})]$ by reaction with $\mathrm{Li}(\mathrm{MeOXL}){ }^{14}$ These reactions are summarized in eq 2.

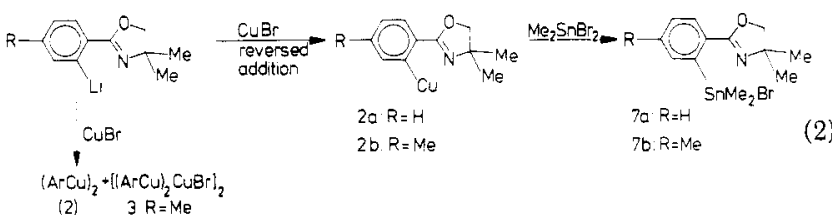

The formation of a mixture of $[\mathrm{Cu}(\mathrm{MeOXL})]$ and $\left[\mathrm{Cu}_{2}(\mathrm{MeOXL})_{2} \mathrm{CuBr}\right]_{2}$ upon addition of $\mathrm{Li}(\mathrm{MeOXL})$ to $\mathrm{CuBr}$ emphasizes the importance of the correct order of addition of the reagents in the synthesis of organocopper compounds. With this order of addition of the reactants there is during the early stages of the reaction an excess of $\mathrm{CuBr}$ present that reacts concurrently with already formed $[\mathrm{Cu}(\mathrm{MeOXL})]$. In previous studies we have shown that arylcopper(I) compounds can easily react with $\mathrm{CuBr}$ either resulting in complexation of $\mathrm{CuBr}^{15}$ or giving rise to biaryl coupling. ${ }^{16}$ The presence of a copper-carbon bond in $2 \mathbf{a}$ and $2 \mathbf{b}$ was indicated by the isolation of the (2-(2-oxazolinyl)aryl)dimethyltin bromide complexes, 7 , from the reaction of these compounds with $\mathrm{Me}_{2} \mathrm{SnBr}_{2} .{ }^{17}$

A remarkable difference was observed in the reactivities of $\mathrm{Li}(\mathrm{OXL})$ and $\mathrm{Li}(\mathrm{MeOXL})$ with $\mathrm{CuBr}\left\{\mathrm{P}(\mathrm{OMe})_{3}\right\}$. Whereas the 1:1 molar reaction of the former with $\mathrm{CuBr}\left\{\mathrm{P}(\mathrm{OMe})_{3}\right\}$ in diethyl ether afforded the pure arylcopper complex $[\mathrm{Cu}(\mathrm{OXL})]$ without coordinated $\mathrm{P}(\mathrm{OMe})_{3}$,

(14) This contrasts with the behavior of previously reported [ $\left.\left.(\mathrm{RCu})_{2}(\mathrm{CuBr})\right\}_{2}\right]\left(\mathrm{R}=\mathrm{C}_{6} \mathrm{H}_{4} \mathrm{NMe}_{2}-2\right)$, which could be converted in a slow reaction with RLi into a pure copper compound: van Koten, G.; Leusink, A. J.: Noltes, J. G. J. Organomet. Chem. 1975, 85, 105.

(15) van Koten, G.; Noltes, J. G. J. Organomet. Chem. 1975, $102,551$.

(16) (a) van Koten, G.; Noltes, J. G. J. Organomet. Chem. 1975, 84, 419. (b) Wehman, E.; van Koten, G.; Knotter, M.; Spelten, H.; Heijdenrijk, D.; Mak, A. N. S.; Stam, C. H. J. Organomet. Chem. 1987, $325,293$.

(17) According to an X-ray structure determination of 7: $\mathrm{Sn}-\mathrm{N}=$ 2.414 (4) $\AA, \mathrm{Sn}-\mathrm{Br}=2.6787$ (8) $\AA, \sum \mathrm{Sn}-\mathrm{C}$ in the trigonal plane $=357.8^{\circ}$ : Wehman, E.; van Koten, G.; Jastrzebski, J. T. B. H., to be submitted for publication. 


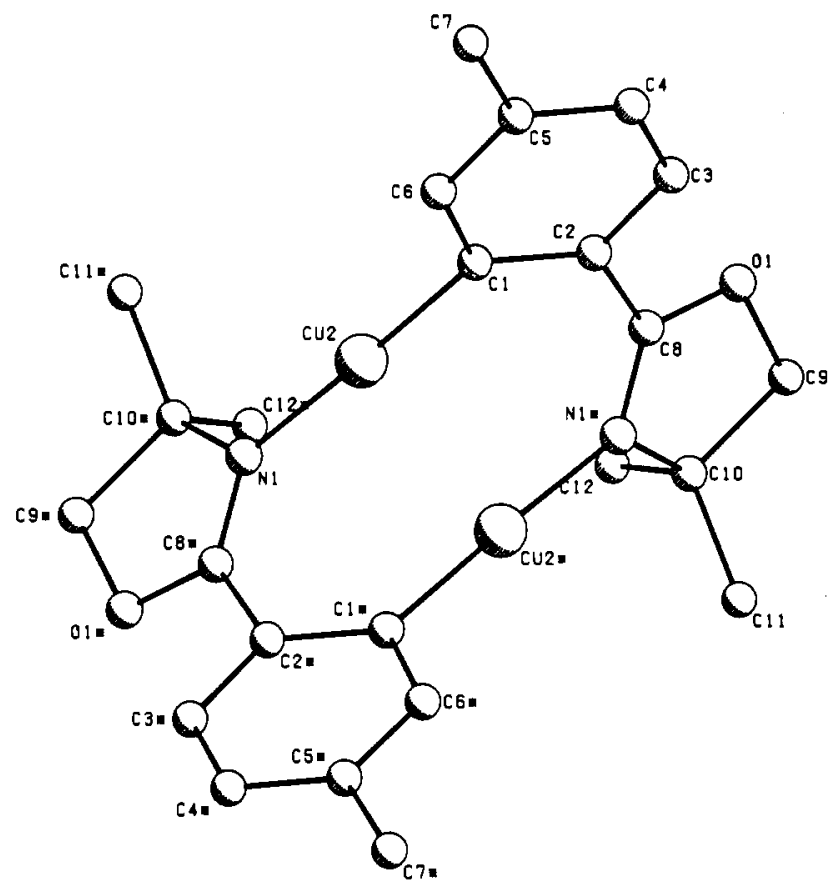

Figure 1. Molecular structure and numbering scheme of $[\mathrm{Cu}-$ $(\mathrm{MeOXL})]_{2}$ (2b). Some relevant bond distances $(\AA): \mathrm{Cu}(2)-\mathrm{C}(1)$ $=1.899(5), \mathrm{Cu}(2)-\mathrm{N}(1)=1.902(4), \mathrm{Cu}(2)-\mathrm{Cu}(2) *=2.4708(9)$, $\mathrm{C}(2)-\mathrm{C}(8)=1.479(7)$, and $\mathrm{N}(1)-\mathrm{C}(8)=1.279(6)$. Some relevant bond angles (deg): $\mathrm{N}(1)-\mathrm{Cu}(2)-\mathrm{C}(1)=177.8(2)$ and $\mathrm{Cu}(2)-\mathrm{N}$ $(1)-C(8)=126.5(4)$.

pure $[\mathrm{Cu}(\mathrm{MeOXL})]$ could not be obtained via this route from $\mathrm{Li}(\mathrm{MeOXL})$. The $100-\mathrm{MHz}{ }^{1} \mathrm{H}$ NMR spectrum in $\mathrm{C}_{6} \mathrm{D}_{6}$ of the product obtained in the latter case indicated clearly the presence of $[\mathrm{Cu}(\mathrm{MeOXL})]$, but it was not possible either to separate this from $\mathrm{P}(\mathrm{OMe})_{3}$ or to isolate a well-defined $\mathrm{P}(\mathrm{OMe})_{3}$-complexed compound (vide infra).

The 2:1 reaction of $\mathrm{Li}(\mathrm{MeOXL})$ with $\mathrm{CuBr}$, shown in eq 3 , afforded a pale yellow, diethyl ether insoluble compound, 4b, whose elemental analytical data pointed to a $\mathrm{CuLi}$ $(\mathrm{MeOXL})_{2}\left(\mathrm{Et}_{2} \mathrm{O}\right)$ stoichiometry, i.e. to the formation of a cuprate that contains coordinated diethyl ether. The

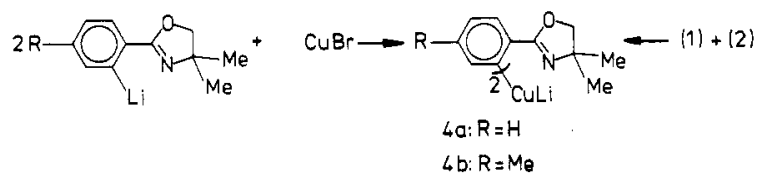

diethyl ether could not be removed from the solid in vacuo, but after dissolution in benzene and evaporation of the benzene, diethyl ether free cuprate was obtained. This was confirmed by the $1: 1$ reaction of $\mathrm{Li}(\mathrm{MeOXL})$ with the corresponding organocopper compound $[\mathrm{Cu}(\mathrm{MeOXL})]$. This reaction afforded a pale yellow compound with essentially the same ${ }^{1} \mathrm{H}$ NMR spectrum as the product 4 obtained via the $\mathrm{RLi} / \mathrm{CuBr}$ route.

The solid (oxazolinylaryl)copper compounds $\mathbf{2 a}$ and $\mathbf{2 b}$ have reasonable oxidation stability which allows them to be handled safely in air for short periods. However, exposure of benzene or diethyl ether solutions of these organocopper compounds to air quickly results in the formation of black precipitates, most likely $\mathrm{Cu}(0)$. The cuprate 4 is thermally less stable than the corresponding organocopper compound $\mathbf{2 b}$. It oxidizes quickly on exposure to air and, moreover, requires storage at low temperature $\left(-20^{\circ} \mathrm{C}\right)$ under an atmosphere of nitrogen to prevent decomposition.

X-ray Crystal Structures of [Cu(MeOXL) $]_{2}(2 b)$ and $\left[\mathrm{Cu}_{2}(\mathrm{MeOXL})_{2} \mathrm{CuBr}\right]_{2}$ (3). The X-ray crystal
Table I. Fractional Coordinates of the Non-Hydrogen Atoms of $\mathrm{Cu}_{2} \mathrm{C}_{24} \mathrm{H}_{28} \mathrm{~N}_{2} \mathrm{O}_{2}, \mathrm{Cu}_{2}(\mathrm{MeOXL})_{2}(2 \mathrm{~b})$

\begin{tabular}{lrlll}
\hline atom & $x$ & \multicolumn{1}{c}{$y$} & \multicolumn{1}{c}{$z$} & \multicolumn{1}{c}{$U_{\text {eq, }} \AA^{2}$} \\
\hline $\mathrm{Cu}(1)$ & $0.26356(4)$ & $0.76939(10)$ & $0.18887(4)$ & $0.0339(3)$ \\
$\mathrm{Cu}(2)$ & $-0.19792(3)$ & $0.4285(1)$ & $0.22324(3)$ & $0.0348(3)$ \\
$\mathrm{C}(1)$ & $-0.3629(2)$ & $0.5579(7)$ & $0.1920(2)$ & $0.033(2)$ \\
$\mathrm{C}(2)$ & $-0.3689(2)$ & $0.5063(7)$ & $0.1183(2)$ & $0.033(2)$ \\
$\mathrm{C}(3)$ & $-0.4174(3)$ & $0.6023(8)$ & $0.0578(3)$ & $0.043(3)$ \\
$\mathrm{C}(4)$ & $-0.4591(3)$ & $0.7638(8)$ & $0.0693(3)$ & $0.049(3)$ \\
$\mathrm{C}(5)$ & $-0.4532(3)$ & $0.8262(8)$ & $0.1410(3)$ & $0.045(3)$ \\
$\mathrm{C}(6)$ & $-0.4067(2)$ & $0.7219(8)$ & $0.2004(3)$ & $0.040(3)$ \\
$\mathrm{C}(7)$ & $-0.4977(4)$ & $1.005(1)$ & $0.1555(4)$ & $0.075(4)$ \\
$\mathrm{C}(8)$ & $-0.3219(2)$ & $0.3492(2)$ & $0.1004(2)$ & $0.034(2)$ \\
$\mathrm{C}(9)$ & $-0.2920(3)$ & $0.1194(10)$ & $0.0253(3)$ & $0.063(4)$ \\
$\mathrm{C}(10)$ & $-0.2330(3)$ & $0.1252(7)$ & $0.1002(3)$ & $0.038(2)$ \\
$\mathrm{C}(11)$ & $-0.1561(3)$ & $0.158(1)$ & $0.0928(4)$ & $0.068(4)$ \\
$\mathrm{C}(12)$ & $-0.2380(4)$ & $0.9395(9)$ & $0.1452(4)$ & $0.067(4)$ \\
$\mathrm{C}(13)$ & $0.1740(2)$ & $0.9030(7)$ & $0.1418(2)$ & $0.035(2)$ \\
$\mathrm{C}(14)$ & $0.1729(3)$ & $1.0676(7)$ & $0.0946(2)$ & $0.041(3)$ \\
$\mathrm{C}(15)$ & $0.1099(3)$ & $1.1823(8)$ & $0.0633(3)$ & $0.047(3)$ \\
$\mathrm{C}(16)$ & $0.0438(3)$ & $1.1293(9)$ & $0.0768(3)$ & $0.053(3)$ \\
$\mathrm{C}(17)$ & $0.0415(3)$ & $0.9645(8)$ & $0.1210(3)$ & $0.048(3)$ \\
$\mathrm{C}(18)$ & $0.1062(2)$ & $0.8568(7)$ & $0.1541(3)$ & $0.036(2)$ \\
$\mathrm{C}(19)$ & $0.1146(4)$ & $0.3651(9)$ & $0.0162(3)$ & $0.069(4)$ \\
$\mathrm{C}(20)$ & $0.3810(2)$ & $0.4554(7)$ & $0.2076(3)$ & $0.036(2)$ \\
$\mathrm{C}(21)$ & $0.4602(3)$ & $0.4420(8)$ & $0.2579(3)$ & $0.048(3)$ \\
$\mathrm{C}(22)$ & $0.4002(2)$ & $0.6930(7)$ & $0.2961(3)$ & $0.035(2)$ \\
$\mathrm{C}(23)$ & $0.3777(4)$ & $0.4792(10)$ & $0.1261(3)$ & $0.062(4)$ \\
$\mathrm{C}(24)$ & $0.3354(3)$ & $0.2788(8)$ & $0.2200(3)$ & $0.048(3)$ \\
$\mathrm{N} 1)$ & $-0.2564(2)$ & $0.3023(6)$ & $0.1363(2)$ & $0.032(2)$ \\
$\mathrm{N}(2)$ & $0.3541(2)$ & $0.6424(6)$ & $0.2354(2)$ & $0.033(2)$ \\
$\mathrm{O}(1)$ & $-0.3506(2)$ & $0.2460(6)$ & $0.0373(2)$ & $0.050(2)$ \\
$\mathrm{O}(2)$ & $0.4632(2)$ & $0.5855(6)$ & $0.3164(2)$ & $0.048(2)$
\end{tabular}

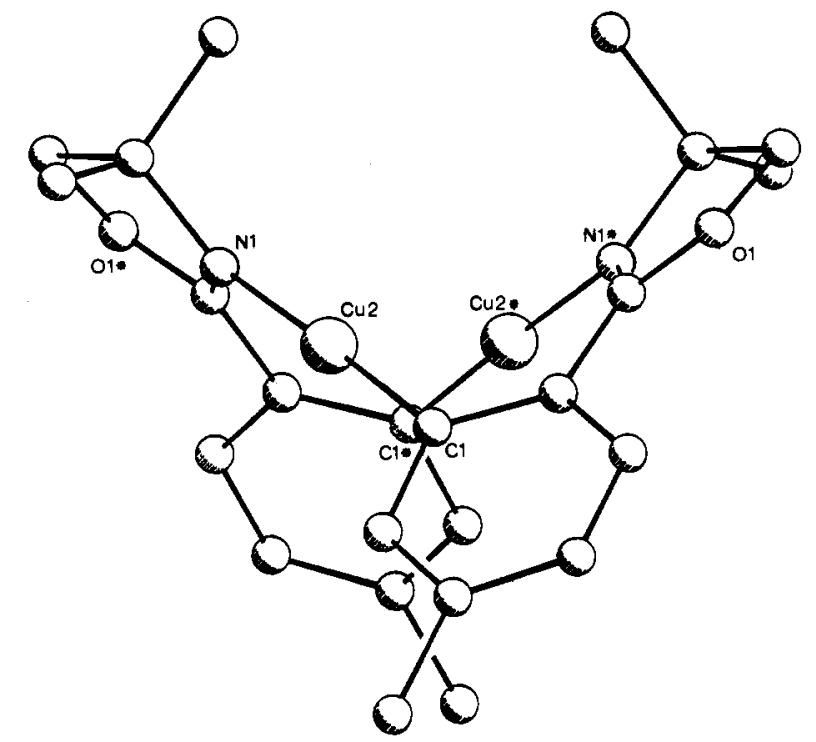

Figure 2. Projection of $2 \mathrm{~b}$ showing the angle of the $\mathrm{C}-\mathrm{Cu}-\mathrm{C}^{\prime}$ axes in the dimer.

structure of $[\mathrm{Cu}(\mathrm{MeOXL})]_{2}$ comprises two independent dimeric arylcopper molecules per unit cell. Figure 1 shows the molecular structure of one of these dimers along with the atomic numbering scheme and some of the relevant distances and bond angles. A complete list of bond distances and angles as well as the corresponding data for the second closely comparable molecule are available as supplementary material. Two oxazolinyl groups act as bidentate bridging units between the two copper atoms, each of which is bonded via a two-center, two-electron bond to $\mathrm{C}$ (ipso) of an aryl ring and to the nitrogen atom of its oxazoline substituent. The $\mathrm{Cu}-\mathrm{C}$ distance $(1.899$ (5) $\AA$ ) is a little shorter than those reported for most arylcopper compounds. ${ }^{7}$ The $\mathrm{Cu}-\mathrm{N}$ distance $(1.902$ (4) $\AA$ ) is in accordance with the value expected for a $\mathrm{Cu}-\mathrm{N}\left(\mathrm{sp}^{2}\right)$ bond. ${ }^{18}$ 
Table II. Fractional Coordinates of the Non-Hydrogen Atoms of $\mathrm{Cu}_{6} \mathrm{C}_{48} \mathrm{H}_{56} \mathrm{~N}_{4} \mathrm{O}_{4} \mathrm{Br}_{29}\left[\mathrm{Cu}_{2}(\mathrm{MeOXL})_{2} \mathrm{CuBr}\right]_{2}$ (3)

\begin{tabular}{|c|c|c|c|c|}
\hline atom & $x$ & $y$ & $z$ & $U_{\text {eq }} \AA^{2}$ \\
\hline $\mathrm{Cu}(1)$ & $0.63185(8)$ & $0.20131(6)$ & $0.72297(9)$ & $0.0338(6)$ \\
\hline $\mathrm{Cu}(2)$ & $0.78299(9)$ & $0.30788(6)$ & $0.80952(10)$ & $0.0355(6)$ \\
\hline $\mathrm{Cu}(3)$ & $0.62663(9)$ & $0.36832(6)$ & $0.81060(10)$ & $0.0358(6)$ \\
\hline $\mathrm{Cu}(4)$ & $0.57655(9)$ & $0.25940(7)$ & $0.91660(10)$ & $0.0371(6)$ \\
\hline $\mathrm{Cu}(5)$ & $0.47908(9)$ & $0.24006(7)$ & $0.70980(10)$ & $0.0389(7)$ \\
\hline $\mathrm{Cu}(6)$ & $0.68780(10)$ & $0.33360(7)$ & $0.62936(10)$ & $0.0397(7)$ \\
\hline $\mathrm{Br}(1)$ & $0.50480(9)$ & $0.29170(7)$ & $0.52265(10)$ & $0.0555(7)$ \\
\hline $\operatorname{Br}(2)$ & $0.76124(8)$ & $0.29838(7)$ & $1.01922(8)$ & $0.0467(6)$ \\
\hline$C(11)$ & $0.4864(7)$ & $0.1336(5)$ & $0.6904(8)$ & $0.037(5)$ \\
\hline$C(12)$ & $0.4304(8)$ & $0.0806(6)$ & $0.7662(8)$ & $0.042(6)$ \\
\hline$C(13)$ & $0.3618(9)$ & $0.0086(6)$ & $0.7195(10)$ & $0.056(7)$ \\
\hline $\mathrm{C}(14)$ & $0.3445(9)$ & $-0.0137(6)$ & $0.6030(10)$ & $0.057(7)$ \\
\hline$C(15)$ & $0.3979(8)$ & $0.0358(6)$ & $0.5242(9)$ & $0.044(6)$ \\
\hline$C(16)$ & $0.4666(7)$ & $0.1058(6)$ & $0.5706(9)$ & $0.043(6)$ \\
\hline$C(17)$ & $0.3777(10)$ & $0.0142(7)$ & $0.3949(10)$ & $0.062(8)$ \\
\hline$C(18)$ & $0.4371(7)$ & $0.0981(5)$ & $0.8906(9)$ & $0.042(6)$ \\
\hline$C(19)$ & $0.3840(14)$ & $0.0740(10)$ & $1.0670(12)$ & $0.113(12)$ \\
\hline $\mathrm{C}(21)$ & $0.7799(7)$ & $0.2076(5)$ & $0.7206(8)$ & $0.039(5)$ \\
\hline $\mathrm{C}(22)$ & $0.8285(7)$ & $0.2081(5)$ & $0.6139(9)$ & $0.043(6)$ \\
\hline$C(23)$ & $0.8907(9)$ & $0.1637(7)$ & $0.5940(11)$ & $0.062(7)$ \\
\hline$C(24)$ & $0.9062(8)$ & $0.1213(6)$ & $0.6700(12)$ & $0.063(8)$ \\
\hline$C(25)$ & $0.8604(8)$ & $0.1182(6)$ & $0.7760(11)$ & $0.056(7)$ \\
\hline$C(26)$ & $0.7979(8)$ & $0.1627(5)$ & $0.7998(9)$ & $0.044(6)$ \\
\hline$C(27)$ & $0.8760(12)$ & $0.0704(9)$ & $0.8680(15)$ & $0.097(12)$ \\
\hline $\mathrm{C}(28)$ & $0.8232(9)$ & $0.2581(6)$ & $0.5257(10)$ & $0.055(7)$ \\
\hline$C(29)$ & $0.8670(23)$ & $0.3030(16)$ & $0.3570(20)$ & $0.214(29)$ \\
\hline$C(31)$ & $0.5350(7)$ & $0.3546(6)$ & $0.9413(8)$ & $0.039(6)$ \\
\hline $\mathrm{C}(32$ & $0.4273(7)$ & $0.3457(6)$ & $0.9445(9)$ & $0.042(6)$ \\
\hline$C(33)$ & $0.3935(8)$ & $0.3852(7)$ & $1.0300(10)$ & $0.063(8)$ \\
\hline$C(34)$ & $0.4626(10)$ & $0.4391(8)$ & $1.1120(10)$ & 0.069 (9) \\
\hline$C(35)$ & $0.5699(9)$ & $0.4527(6)$ & $1.1105(10)$ & $0.057(7)$ \\
\hline$C(36)$ & $0.6028(8)$ & $0.4088(6)$ & $1.0276(9)$ & $0.045(6)$ \\
\hline$C(37)$ & $0.6460(10)$ & $0.5086(8)$ & $1.2010(12)$ & $0.079(9)$ \\
\hline $\mathrm{C}(38)$ & $0.3454(8)$ & $0.2920(6)$ & $0.8625(10)$ & $0.047(6)$ \\
\hline$C(39)$ & $0.1753(9)$ & $0.2228(8)$ & $0.8060(12)$ & $0.077(9)$ \\
\hline $\mathrm{C}(41)$ & $0.7218(7)$ & $0.4405(5)$ & 0.7138 & $0.035(5)$ \\
\hline $\mathrm{C}(42)$ & $0.8248(8)$ & $0.4913(5)$ & $0.7486(7)$ & $0.036(5)$ \\
\hline$C(43)$ & $0.8536(8)$ & $0.5657(6)$ & $0.7340(10)$ & $0.055(7)$ \\
\hline$C(44)$ & $0.7870(10)$ & $0.5955(6)$ & $0.6830(11)$ & $0.066(8)$ \\
\hline$C(45)$ & $0.6856(9)$ & $0.5496(6)$ & 0.6478 (9) & $0.052(7)$ \\
\hline$C(46)$ & $0.6545(9)$ & $0.4737(6)$ & $0.6612(9)$ & $0.051(7)$ \\
\hline$C(47)$ & $0.6090(12)$ & $0.5804(7)$ & $0.5910(14)$ & $0.092(10)$ \\
\hline$C(48)$ & $0.9082(7)$ & $0.4653(5)$ & $0.7929(8)$ & $0.037(5)$ \\
\hline C(49) & $1.0733(8)$ & $0.4905(6)$ & $0.8656(10)$ & $0.051(6)$ \\
\hline$C(100)$ & $0.4790(10)$ & 0.1449 (7) & $1.0787(9)$ & $0.065(7)$ \\
\hline$C(110)$ & $0.5770(14)$ & $0.1350(9)$ & $1.1290(12)$ & $0.098(12)$ \\
\hline$C(120)$ & $0.4610(13)$ & $0.2128(10)$ & $1.1470(11)$ & $0.094(11)$ \\
\hline$C(200)$ & $0.8063(9)$ & $0.3441(7)$ & $0.4156(9)$ & $0.058(7)$ \\
\hline$C(210)$ & $0.7100(15)$ & $0.3410(14)$ & $0.3450(13)$ & $0.141(17)$ \\
\hline$C(220)$ & $0.8790(19)$ & $0.4220(10)$ & $0.4520(14)$ & $0.142(15)$ \\
\hline$C(300)$ & $0.2424(8)$ & $0.2135(8)$ & $0.7120(12)$ & $0.069(8)$ \\
\hline $\mathrm{C}(310)$ & $0.2230(10)$ & $0.1349(9)$ & $0.6650(18)$ & $0.113(12)$ \\
\hline$C(320)$ & $0.2340(12)$ & 0.2650 (11) & $0.6130(13)$ & $0.100(12)$ \\
\hline$C(400)$ & $1.0172(8)$ & $0.4050(6)$ & $0.8321(8)$ & $0.042(6)$ \\
\hline$C(410)$ & $1.0297(9)$ & $0.3625(7)$ & $0.9290(11)$ & $0.065(8)$ \\
\hline$C(420)$ & $1.0513(8)$ & $0.3776(7)$ & $0.7195(10)$ & $0.060(7)$ \\
\hline$N(1)$ & $0.4924(7)$ & $0.1603(5)$ & $0.9543(7)$ & $0.046(5)$ \\
\hline $\mathrm{N}(2)$ & $0.7778(7)$ & 0.3049 (5) & $0.5207(7)$ & $0.050(5)$ \\
\hline$N(3)$ & $0.3524(6)$ & $0.2502(5)$ & $0.7725(8)$ & $0.048(5)$ \\
\hline $\mathrm{N}(4)$ & $0.9069(5)$ & $0.4005(4)$ & $0.8081(6)$ & $0.035(4)$ \\
\hline $\mathrm{O}(1)$ & $0.3763(7)$ & $0.0421(4)$ & $0.9484(7)$ & $0.076(6)$ \\
\hline $\mathrm{O}(2)$ & $0.8790(8)$ & $0.2513(6)$ & $0.4317(7)$ & $0.087(7)$ \\
\hline $\mathrm{O}(3)$ & $0.2436(5)$ & $0.2860(5)$ & $0.8844(8)$ & $0.072(5)$ \\
\hline $\mathrm{O}(4)$ & $1.0042(5)$ & $0.5226(4)$ & $0.8136(6)$ & $0.047(4)$ \\
\hline
\end{tabular}

The copper atoms are almost linearly coordinated $(\mathrm{N}-$ $\left.\mathrm{Cu}-\mathrm{C}=177.8(2)^{\circ}\right)$. From Figure 2 it can be clearly seen that the $\mathrm{N}(1)-\mathrm{Cu}(2)-\mathrm{C}(1)$ and $\mathrm{N}\left(1^{*}\right)-\mathrm{Cu}\left(2^{*}\right)-\mathrm{C}\left(1^{*}\right)$ axes are not parallel and that the oxazoline substituent is rotated out of the plane of the aryl group to which it is bonded.

(18) Spek, A. L.; Duisenberg, A. J. M.; van Stein, G. C.; van Koten, G. Acta Crystallogr., Sect. C: Cryst. Struct. Commun, 1985, C41, 374.

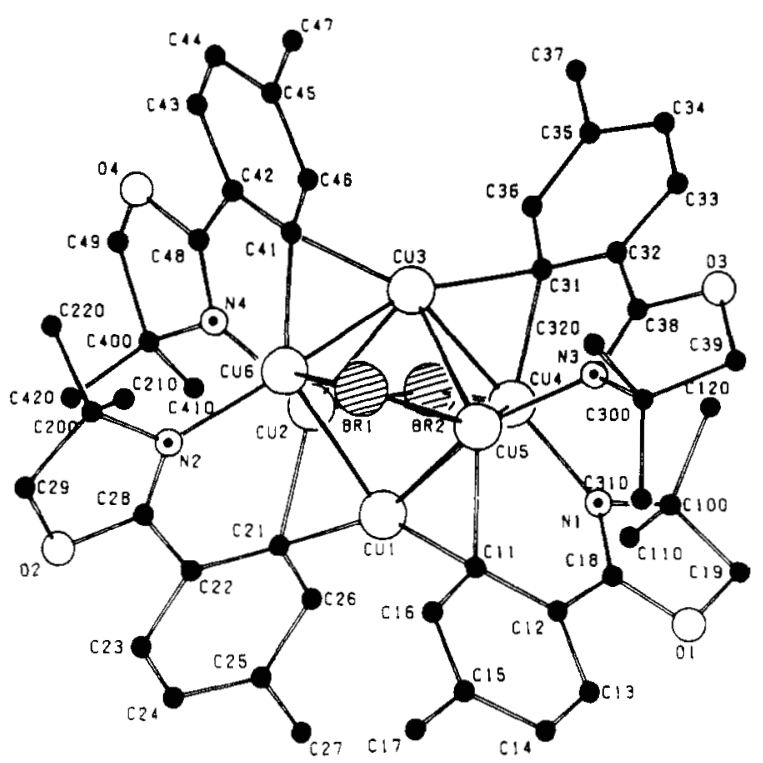

Figure 3. Molecular structure and numbering scheme of $\left[\mathrm{Cu}_{2}-\right.$ $\left.(\mathrm{MeOXL})_{2} \mathrm{CuBr}\right]_{2}(3)$.

Table III. Some Relevant Bond Distances $(\AA)$ and Angles (deg) with Standard Deviations in Parentheses of $\left[\mathrm{Cu}_{2}(\mathrm{MeOXL})_{2} \mathrm{CuBr}\right]_{2}(3)$

\begin{tabular}{llll} 
& \multicolumn{4}{c}{ Bond Distances } \\
$\mathrm{Cu}(1)-\mathrm{Cu}(2)$ & $2.434(5)$ & $\mathrm{Cu}(4)-\mathrm{Br}(2)$ & $2.570(7)$ \\
$\mathrm{Cu}(1)-\mathrm{Cu}(4)$ & $2.678(5)$ & $\mathrm{Cu}(4)-\mathrm{N}(1)$ & $1.948(9)$ \\
$\mathrm{Cu}(1)-\mathrm{Cu}(5)$ & $2.451(5)$ & $\mathrm{Cu}(4)-\mathrm{C}(31)$ & $2.090(10)$ \\
$\mathrm{Cu}(1)-\mathrm{Cu}(6)$ & $2.728(5)$ & $\mathrm{Cu}(5)-\mathrm{Cu}(6)$ & $3.033(5)$ \\
$\mathrm{Cu}(1)-\mathrm{C}(11)$ & $1.952(9)$ & $\mathrm{Cu}(5)-\mathrm{Br}(1)$ & $2.470(4)$ \\
$\mathrm{Cu}(1)-\mathrm{C}(21)$ & $1.985(9)$ & $\mathrm{Cu}(5)-\mathrm{N}(3)$ & $1.972(8)$ \\
$\mathrm{Cu}(2)-\mathrm{Cu}(3)$ & $2.763(6)$ & $\mathrm{Cu}(5)-\mathrm{C}(11)$ & $2.068(8)$ \\
$\mathrm{Cu}(2)-\mathrm{Cu}(4)$ & $3.012(6)$ & $\mathrm{Cu}(6)-\mathrm{Br}(1)$ & $2.565(5)$ \\
$\mathrm{Cu}(2)-\mathrm{Cu}(6)$ & $2.600(5)$ & $\mathrm{Cu}(6)-\mathrm{N}(2)$ & $1.974(8)$ \\
$\mathrm{Cu}(2)-\mathrm{Br}(2)$ & $2.493(5)$ & $\mathrm{Cu}(6)-\mathrm{C}(41)$ & $2.071(8)$ \\
$\mathrm{Cu}(2)-\mathrm{N}(4)$ & $1.980(8)$ & $\mathrm{N}(1)-\mathrm{C}(18)$ & $1.302(11)$ \\
$\mathrm{Cu}(2)-\mathrm{C}(21)$ & $2.079(9)$ & $\mathrm{N}(2)-\mathrm{C}(28)$ & $1.261(12)$ \\
$\mathrm{Cu}(3)-\mathrm{Cu}(4)$ & $2.432(6)$ & $\mathrm{N}(3)-\mathrm{C}(38)$ & $1.286(11)$ \\
$\mathrm{Cu}(3)-\mathrm{Cu}(5)$ & $2.694(5)$ & $\mathrm{N}(4)-\mathrm{C}(48)$ & $1.265(10)$ \\
$\mathrm{Cu}(3)-\mathrm{Cu}(6)$ & $2.426(5)$ & $\mathrm{C}(12)-\mathrm{C}(18)$ & $1.449(11)$ \\
$\mathrm{Cu}(3)-\mathrm{C}(31)$ & $1.993(9)$ & $\mathrm{C}(22)-\mathrm{C}(28)$ & $1.486(11)$ \\
$\mathrm{Cu}(3)-\mathrm{C}(41)$ & $2.011(9)$ & $\mathrm{C}(32)-\mathrm{C}(38)$ & $1.462(12)$ \\
$\mathrm{Cu}(4)-\mathrm{Cu}(5)$ & $2.634(5)$ & $\mathrm{C}(42)-\mathrm{C}(48)$ & $1.484(12)$
\end{tabular}

Bond Angles

$\mathrm{C}(11)-\mathrm{Cu}(1)-\mathrm{C}(21) \quad 143.9(4) \quad \mathrm{Cu}(3)-\mathrm{C}(31)-\mathrm{Cu}(4) \quad 73.1(5)$ $\mathrm{C}(31)-\mathrm{Cu}(3)-\mathrm{C}(41) \quad 147.1(4) \quad \mathrm{C}(32)-\mathrm{C}(31)-\mathrm{C}(36) \quad 114.6(9)$ $\mathrm{Cu}(1)-\mathrm{C}(11)-\mathrm{Cu}(5) \quad 75.1(5) \quad \mathrm{Cu}(3)-\mathrm{C}(41)-\mathrm{Cu}(6) \quad 72.9(5)$ $\mathrm{C}(12)-\mathrm{C}(11)-\mathrm{C}(16) \quad 114.5(8) \quad \mathrm{C}(42)-\mathrm{C}(41)-\mathrm{C}(46) \quad 114.4(10)$ $\mathrm{Cu}(1)-\mathrm{C}(21)-\mathrm{Cu}(2) \quad 73.5(5) \quad \mathrm{Cu}(5)-\mathrm{Br}(1)-\mathrm{Cu}(6) \quad 74.04(20)$ $\mathrm{C}(22)-\mathrm{C}(21)-\mathrm{C}(26) \quad 117.2(9) \quad \mathrm{Cu}(2)-\mathrm{Br}(2)-\mathrm{Cu}(4) \quad 73.0(3)$

The structure of $\left[\mathrm{Cu}_{2}(\mathrm{MeOXL})_{2} \mathrm{CuBr}\right]_{2}$, whose X-ray molecular structure and the atomic numbering scheme are shown in Figure 3 (see for some relevant bond distances and angles Table III), is of a different type. ${ }^{8}$ This complex that can be thought of as a dimer of $\mathrm{Cu}_{2}(\mathrm{MeOXL})_{2} \mathrm{CuBr}$ has a structure built up of six $\mathrm{Cu}$ atoms positioned at the apices of a distorted octahedron. Two opposite equatorial edges of the octahedron are each bridged by a single bromine atom. The four trigonal faces that contain the two other equatorial edges are each bridged by one 2-(2-oxazolinyl)aryl ligand. The C(ipso) atom of each ligand bridges a copper(apical)-copper(equatorial) bond in a three-center, two-electron interaction and the oxazoline $\mathrm{N}$ atom binds to the third copper atom of the trigonal face such that each equatorial $\mathrm{Cu}$ atom is structurally equivalent. For these ligands it is worth noting that there is a dihedral angle of $31^{\circ}$ between the planes of the oxazoline substituent and the aryl rings. The $\mathrm{C}-\mathrm{C}$ bridged $\mathrm{Cu}-\mathrm{Cu}$ 
Table IV. 'H NMR Data ${ }^{a}$ of the Aryloxazoline Complexes

\begin{tabular}{|c|c|c|c|c|c|c|c|}
\hline compd & solv & temp, K & $\mathrm{OXL} \mathrm{CH}_{3}$ & Aryl $\mathrm{CH}_{3}$ & oxazoline $\mathrm{CH}_{2}$ & aryl protons & miscellaneous \\
\hline$\overline{\mathrm{OXL}-\mathrm{H}}$ & $\mathrm{C}_{6} \mathrm{D}_{6}$ & $\mathrm{AT}$ & $1.17(\mathrm{~s})$ & & $3.71(\mathrm{~s})$ & $\begin{array}{c}8.18\left(\mathrm{~m}, 2 \mathrm{H}, \mathrm{H}_{2}+\mathrm{H}_{6}\right), 7.00(\mathrm{~m}, 3 \\
\left.\mathrm{H}, \mathrm{H}_{3}+\mathrm{H}_{4}+\mathrm{H}_{5}\right)\end{array}$ & \\
\hline \multirow[t]{2}{*}{$\mathrm{MeOXL}-\mathrm{H}$} & $\mathrm{C}_{6} \mathrm{D}_{6}$ & AT & $1.18(\mathrm{~s})$ & $1.96(\mathrm{~s})$ & $3.70(\mathrm{~s})$ & $\begin{array}{l}8.20\left(\mathrm{~d}, 2 \mathrm{H}, \mathrm{H}_{2}+\mathrm{H}_{6},{ }^{2} J_{\mathrm{HH}}=8 \mathrm{~Hz}\right) \\
6.90\left(\mathrm{~d}, 2 \mathrm{H}, \mathrm{H}_{3}+\mathrm{H}_{5},{ }^{2} J_{\mathrm{HH}}=8\right. \\
\mathrm{Hz})\end{array}$ & \\
\hline & $\mathrm{CDCl}_{3}$ & AT & $1.33(\mathrm{~s})$ & $2.33(\mathrm{~s})$ & $4.02(\mathrm{~s})$ & $\begin{array}{l}7.80\left(\mathrm{~d}, 2 \mathrm{H}, \mathrm{H}_{2}+\mathrm{H}_{6},{ }^{2} J_{\mathrm{HH}}=8 \mathrm{~Hz}\right) \\
7.13\left(\mathrm{~d}, 2 \mathrm{H}, \mathrm{H}_{3}+\mathrm{H}_{5},{ }^{2} J_{\mathrm{HH}}=8\right. \\
\mathrm{Hz})\end{array}$ & \\
\hline $5 a(2-O X L-D)$ & $\mathrm{CDCl}_{3}$ & $\mathrm{AT}$ & $1.38(\mathrm{~s})$ & & $4.06(\mathrm{~s})$ & $\begin{array}{l}7.90\left(\mathrm{~m}, 1 \mathrm{H}, \mathrm{H}_{6}\right), 7.20,\left(\mathrm{~b}, 3 \mathrm{H}, \mathrm{H}_{3}\right. \\
\left.+\mathrm{H}_{4}+\mathrm{H}_{5}\right)\end{array}$ & \\
\hline 5b (2-MeOXL-D) & $\mathrm{CDCl}_{3}$ & $\mathrm{AT}$ & $1.35(\mathrm{~s})$ & $2.35(\mathrm{~s})$ & $4.03(\mathrm{~s})$ & $\begin{array}{l}7.76\left(\mathrm{~d}, 1 \mathrm{H}, \mathrm{H}_{6},{ }^{2} J_{\mathrm{HH}}=8 \mathrm{~Hz}\right), 7.10 \\
\left(\mathrm{~m}, 2 \mathrm{H}, \mathrm{H}_{3}+\mathrm{H}_{5}\right)\end{array}$ & \\
\hline $6\left(2-\mathrm{MeOXL}_{-} \mathrm{SiMe}_{3}\right)$ & tol- $-d_{8}$ & $233 \mathrm{~K}$ & $1.13(\mathrm{~s})$ & $2.09(\mathrm{~s})$ & $3.57(\mathrm{~s})$ & $\begin{array}{l}8.17\left(\mathrm{~d}, 1 \mathrm{H}, \mathrm{H}_{6}, J_{\mathrm{HH}}=8 \mathrm{~Hz}\right), 7.60 \\
\left(\mathrm{~s}, 1 \mathrm{H}, \mathrm{H}_{3}\right), 6.93\left(\mathrm{~d}, 1 \mathrm{H}, \mathrm{H}_{5}\right. \\
\left.2 J_{\mathrm{HH}}=8 \mathrm{~Hz}\right)\end{array}$ & $0.60\left(\mathrm{~s}, 9 \mathrm{H}, \mathrm{SiMe}_{3}\right)$ \\
\hline 7b $\left(\mathrm{MeOXL}-\mathrm{SnMe} \mathrm{e}_{2} \mathrm{Br}\right)$ & $\mathrm{CDCl}_{3}$ & AT & $1.22(\mathrm{~s})$ & $2.25(\mathrm{~s})$ & $4.20(\mathrm{~s})$ & $\begin{array}{l}8.03\left(\mathrm{~b}, 1 \mathrm{H}, \mathrm{H}_{3}\right), 7.43\left(\mathrm{~d}, 1 \mathrm{H}, \mathrm{H}_{6}\right. \\
\left.2 J_{\mathrm{HH}}=8 \mathrm{~Hz}\right), 7.10\left(\mathrm{~d}, 1 \mathrm{H}, \mathrm{H}_{5}\right. \\
\left.2 J_{\mathrm{HH}}=8 \mathrm{~Hz}\right)\end{array}$ & $\begin{array}{l}0.73\left(\mathrm{~s}, 6 \mathrm{H}, \mathrm{SnMe}_{2},\right. \\
\left.{ }^{2} J_{\mathrm{Sn}-\mathrm{H}}=76 \mathrm{~Hz}\right)\end{array}$ \\
\hline \multirow[t]{2}{*}{ lb $(\mathrm{Li}(\mathrm{MeOXL}))$} & tol- $d_{B}$ & $348 \mathrm{~K}$ & $0.66(\mathrm{~s})^{b}$ & $2.21(\mathrm{~s})$ & $3.62(\mathrm{~s})$ & $\begin{array}{l}8.30\left(\mathrm{~s}, 1 \mathrm{H}, \mathrm{H}_{3}\right), 7.93\left(\mathrm{~d}, 1 \mathrm{H}, \mathrm{H}_{6}\right. \\
\left.2 J_{\mathrm{HH}}=7 \mathrm{~Hz}\right), 6.98\left(\mathrm{~d}, 1 \mathrm{H}, \mathrm{H}_{5}\right. \\
\left.2 J_{\mathrm{HH}}=7 \mathrm{~Hz}\right)\end{array}$ & \\
\hline & & $298 \mathrm{~K}$ & $\begin{array}{l}0.49(\mathrm{~s}, 3 \mathrm{H}) \\
\quad 0.82(\mathrm{~s}, 3 \mathrm{H})\end{array}$ & $2.22(\mathrm{~s})$ & $\begin{array}{l}3.43(\mathrm{~d}, 1 \mathrm{H}) \\
3.73(\mathrm{~d}, \mathrm{I} \mathrm{H}) \\
2 J_{\mathrm{HH}}=7 \mathrm{~Hz}\end{array}$ & $\begin{array}{l}8.13\left(\mathrm{~d}, 1 \mathrm{H}, \mathrm{H}_{6},{ }^{2} J_{\mathrm{HH}}=8 \mathrm{~Hz}\right), 8.37 \\
\left(\mathrm{~s}, 1 \mathrm{H}, \mathrm{H}_{3}\right), 6.92\left(\mathrm{~d}, 1 \mathrm{H}, \mathrm{H}_{5}\right. \\
\left.2 J_{\mathrm{HH}}=8 \mathrm{~Hz}\right)\end{array}$ & \\
\hline $\mathbf{2 a}(\mathrm{Cu}(\mathrm{OXL}))$ & tol- $d_{8}$ & $298 \mathrm{~K}$ & $1.08(\mathrm{~s})$ & & $3.58(\mathrm{~s})$ & $\begin{array}{l}8.18+7.73\left(\mathrm{br} \mathrm{m}, 2 \mathrm{H}, \mathrm{H}_{3}+\mathrm{H}_{6}\right) \\
7.00-7.40\left(\mathrm{br} \mathrm{m}, 2 \mathrm{H}, \mathrm{H}_{4}+\mathrm{H}_{5}\right)\end{array}$ & \\
\hline \multirow[t]{2}{*}{ 2b $(\mathrm{Cu}(\mathrm{MeOXL}))$} & $\begin{array}{l}\text { tol- } d_{8} \\
\text { tol- } d_{8}\end{array}$ & $298 \mathrm{~K}$ & $1.07(\mathrm{~s})$ & $2.25(\mathrm{~s})$ & $\begin{array}{l}3.20-3.70(\mathrm{~b}) \\
3.60(\mathrm{~s})\end{array}$ & $\begin{array}{l}8.0-8.15\left(\mathrm{br} \mathrm{m}, 2 \mathrm{H}, \mathrm{H}_{3}+\mathrm{H}_{5}\right), 6.99 \\
\left(\mathrm{~d}, 1 \mathrm{H}, \mathrm{H}_{6},{ }^{2} J_{\mathrm{HH}}=8 \mathrm{~Hz}\right)\end{array}$ & \\
\hline & tol- $-d_{8}$ & $213 \mathrm{~K}$ & $0.50-1.50^{\mathrm{c}}$ & $2.25-2.50$ & $3.00-3.50$ (b) & $\begin{array}{l}7.50-8.50\left(\mathrm{br} m, \mathrm{H}_{6}\right), 7.00-7.40(\mathrm{br} \\
\left.\quad \mathrm{m}, 2 \mathrm{H}, \mathrm{H}_{4}+\mathrm{H}_{5}\right)\end{array}$ & \\
\hline $3\left(\mathrm{Cu}_{6}(\mathrm{MeOXL})_{4} \mathrm{Br}_{2}\right)$ & $\mathrm{C}_{6} \mathrm{D}_{6}$ & AT & $0.68-1.60^{c}$ & $1.96(\mathrm{~s})$ & $3.49(\mathrm{~s})$ & $\begin{array}{l}8.89\left(\mathrm{~s}, 1 \mathrm{H}, \mathrm{H}_{3}\right), 8.21\left(\mathrm{~d}, 1 \mathrm{H}, \mathrm{H}_{6}\right. \\
\left.{ }^{2} J_{\mathrm{HH}}=8 \mathrm{~Hz}\right), 6.90\left(\mathrm{~d}, 1 \mathrm{H}, \mathrm{H}_{6}\right. \\
\left.{ }^{2} J_{\mathrm{HH}}=8 \mathrm{~Hz}\right)\end{array}$ & \\
\hline $4\left(\mathrm{CuLi}(\mathrm{MeOXL})_{2}\right)$ & tol- $d_{8}$ & $298 \mathrm{~K}$ & $\begin{array}{l}0.70(\mathrm{~s}, 3 \mathrm{H}) \\
\quad 0.91(\mathrm{~s}, 3 \mathrm{H})\end{array}$ & $2.47(\mathrm{~s})$ & $\begin{array}{l}3.49(\mathrm{~d}, 1 \mathrm{H}) \\
\quad 3.62(\mathrm{~d}, 1 \mathrm{H}) \\
2 J_{\mathrm{HH}}=8 \mathrm{~Hz}\end{array}$ & $\begin{array}{c}8.31\left(\mathrm{~b}, 1 \mathrm{H}, \mathrm{H}_{3}\right), 7.75\left(\mathrm{~d}, 1 \mathrm{H}, \mathrm{H}_{6}\right. \\
\left.{ }^{2} J_{\mathrm{HH}}=8 \mathrm{~Hz}\right), 7.10\left(\mathrm{~b}, 1 \mathrm{H}, \mathrm{H}_{3}\right)^{d}\end{array}$ & \\
\hline
\end{tabular}

${ }^{a} \delta$ in parts per million relative to TMS (internal standard); AT = ambient temperature. ${ }^{b}$ Coalescence occurred at $318 \mathrm{~K}$. 'Several peaks were observed in this region.

edge distance of 2.436 (3) $\AA$ agrees well with values for other polynuclear arylcopper compounds. ${ }^{7}$ The $\mathrm{Cu}-\mathrm{N}$ distance (1.968 (7) $\AA$ (mean)) is in the range found for $\mathrm{Cu}(\mathrm{I})-\mathrm{N}\left(\mathrm{sp}^{2}\right)$ bonds ${ }^{18}$ but is significantly shorter than found in compounds examined previously $(2.2-2.3 \AA) .^{19}$ Compared to the distances found in $[\mathrm{Cu}(\mathrm{MeOXL})]_{2}$, the $\mathrm{Cu}-\mathrm{N}$ and $\mathrm{Cu}-\mathrm{C}$ bonds in $\left[\mathrm{Cu}_{2}(\mathrm{MeOXL})_{2} \mathrm{CuBr}\right]_{2}$ are a little elongated. However, the change of a two-center, two-electron copper-carbon bond in $[\mathrm{Cu}(\mathrm{MeOXL})]_{2}$ to a three-center, two-electron bond in $\left[\mathrm{Cu}_{2}(\mathrm{MeOXL})_{2} \mathrm{CuBr}\right]_{2}$ did not significantly influence the atomic distances in the aryl group. For example, the $\mathrm{C}(2)-\mathrm{C}(8)$ bond length in $[\mathrm{Cu}(\mathrm{MeOXL})]_{2}$ of $1.479(7) \AA$ and $\mathrm{C}(12)-\mathrm{C}(18)$ distance in $\left[\mathrm{Cu}_{2}(\mathrm{MeOXL})_{2} \mathrm{CuBr}\right]_{2}$ of $1.449(7) \AA$ are both indicative of delocalized double-bond character.

The structural features of the dinuclear arylcopper compound $[\mathrm{Cu}(\mathrm{MeOXL})]_{2}$ are different from those usually found in arylcopper compounds. Generally organocopper compounds are characterized by (i) being highly aggregated species (tetrameric, ${ }^{16 b, 19 b, c, 20,21}$ pentameric, ${ }^{20}$ or octameric ${ }^{22}$ ) and (ii) having copper-carbon bonds involved in multicenter bonding of a three-center, two-electron type. ${ }^{7}$ Only

(19) (a) Guss, J. M.; Mason, R.; Thomas, K. M.; van Koten, G.; Noltes, J. G. J. Organomet. Chem. 1972, 40, C79. (b) Guss, J. M.; Mason, R.; Sotofte, I.; van Koten, G.; Noltes, J. G. J. Chem. Soc. Chem. Commun. 1972, 446. (c) Noltes, J. G.; ten Hoedt, R. W. M.; van Koten, G.; Spek, A. L.; Schoone, J. C. J. Organomet. Chem. 1982, 225, 365.

(20) Gambarotta, S.; Floriani, C.; Chiesi-Villa, A.; Guastini, C. J. Chem. Soc., Chem. Commun. 1983, 1156.

(21) Jarvis, J. A. J.; Pearce, R.; Lappert, M. F. J. Chem. Soc., Dalton Trans. 1977, 999.

(22) (a) Cairncross, A.; Shepard, W. A. J. Am. Chem. Soc. 1971, 93 247. (b) Camus, A.; Marsich, N.; Nardin, G.; Randaccio, L. J. Organomet. Chem. 1979, 174, 121. two earlier examples of dinuclear organocopper compounds characterized by $\mathrm{X}$-ray diffraction studies are known, viz., $\left[\left\{\mathrm{Me}_{2} \mathrm{P}\left(\mathrm{CH}_{2}\right)_{2} \mathrm{Cu}_{2}\right](\mathbf{1 0})^{23 \mathrm{a}}\right.$ and $\left[\left(2-\left(\mathrm{Me} \mathrm{Si}_{3}\right)_{2} \mathrm{CH}(\mathrm{Cu}) \mathrm{C}_{5} \mathrm{H}_{4} \mathrm{~N}\right\}_{2}\right]$ (11), ${ }^{23 \mathrm{~b}}$ and the structure of $2 \mathrm{~b}$ shows a resemblance to both of these. In 10 and 11 the organo group is bonded to the copper atoms in a bidentate bridging mode with two-center, two-electron copper-carbon bonds. Due to the different number of atoms involved in the $\mathrm{Cu} \ldots \mathrm{Cu}$ bridge, the aromatic rings in 11 are coplanar, whereas in $\mathbf{2 b}$ these are staggered. Despite this difference as well as the difference in bite of the bridging ligands, the resemblance in the geometry around the copper atoms is striking. $2 \mathrm{~b}(11)$ : $\mathrm{Cu}-\mathrm{C}=1.899$ (5) (1.950 (4)) $\AA, \mathrm{Cu}-\mathrm{N}=1.902$ (4) (1.910 (3)) $\AA, \mathrm{Cu}-\mathrm{Cu}=2.4708$ (9) $(2.412(1) \AA)$, and $\mathrm{N}-\mathrm{Cu}-\mathrm{C}=$ $177.8(2)^{\circ}\left(178.0(5)^{\circ}\right)$. With $[\mathrm{Cu}(\mathrm{MeOXL})]_{2}$ the formation of a dimeric structure, instead of a higher aggregate usually found for arylcopper species, can be ascribed to the special requirements of the oxazolinylaryl ligand, namely, (i) the orientation of the lone pair of the $\mathrm{N}$ heteroatom and (ii) the steric bulk of the oxazoline substituent of the aryl system. These requirements make its bonding properties completely different from those of related ligands such as $\mathrm{C}_{6} \mathrm{H}_{4} \mathrm{CH}_{2} \mathrm{NMe}_{2}-2$ or $\mathrm{C}_{6} \mathrm{H}_{4} \mathrm{NMe}_{2}-2$. The orientation of the $\mathrm{N}\left(\mathrm{sp}^{2}\right)$ lone pair only allows a strong $\mathrm{N}$-coordination to the copper atom when the oxazolinylaryl acts as a bidentate bridging group. This ligand is, however, not suited for $\mathrm{C}, \mathrm{N}$-chelate bonding because this would require considerable bending of the $\mathrm{C}=\mathrm{C}-\mathrm{C}=\mathrm{N}$ skeleton. This restriction together with the steric bulk of the oxazoline

(23) (a) Nardin, G.; Randaccio, L.; Zangrando, E. J. Organomet. Chem. 1974, 74, C23. (b) Papasergio, R. I.; Raston, C. L.; White, A. H. J. Chem. Soc., Chem. Commun. 1983, 1419. 


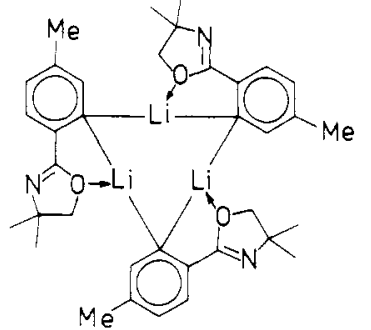

Figure 4. Proposed structure for $[\mathrm{Li}(\mathrm{MeOXL})]_{3}(\mathbf{l b})$.

group prevents the formation of higher order aggregates, and a dimeric compound results. Replacement of a 2-(2oxazolinyl)aryl group by a stereochemically less demanding $\mathrm{Br}$ atom allows higher aggregation, as is reflected in the formation of the hexanuclear structure of $\left[\mathrm{Cu}_{2^{-}}\right.$ $\left.(\mathrm{MeOXL})_{2} \mathrm{CuBr}\right]_{2}$.

Structures in Solution of $[\mathrm{Li}(\mathrm{MeOXL})]_{3}(\mathbf{l b})$ and $[\mathrm{Cu}(\mathrm{MeOXL})]_{2}$ (2b). At $298 \mathrm{~K}$ the $100-\mathrm{MHz}^{1} \mathrm{H}$ NMR spectrum of $\mathrm{Li}(\mathrm{MeOXL})(1 \mathrm{~b})$ in toluene- $d_{8}$ showed diastereotopic signals for the MeOXL Me groups (doublet) and the $\mathrm{MeOXL} \mathrm{CH}_{2}$ protons (AB pattern). At $318 \mathrm{~K}$ these two sets of signals each coalesced to one resonance at averaged positions of the diastereotopic signals, thus indicating that a fluxional process was occurring. It was established independently that the ${ }^{1} \mathrm{H}$ NMR spectra of the corresponding hydrocarbon MeOXL-H and the trimethylsilyl compound 6 were temperature-independent, so excluding the possibility that the fluxional process for $\mathrm{Li}(\mathrm{MeOXL})$ originates from ring conformations of the oxazoline ring. Consequently, the fluxionality can most likely be ascribed either to the presence of an equilibrium between a coordinated and uncoordinated oxazoline substituent or to interaggregate exchange processes. Both processes are now well-documented for this type of aryllithium compound with an heteroatom containing ortho substituent. $^{24}$

On the basis of the NMR spectra obtained it is not possible to ascertain whether it is the $\mathrm{O}$ - or $\mathrm{N}$-donor site of the oxazoline which coordinates, though probably this will be the $\mathrm{O}$ site. The presence of $\mathrm{O}$-coordination could explain why BuLi does not add to the $\mathrm{CN}$ double bond in hexane (see eq 1); coordination to the $\mathrm{N}$ site would activate this bond toward addition. Cryoscopic molecular weight determinations on solutions of $\mathrm{Li}(\mathrm{MeOXL})$ in benzene gave values that were effectively concentration independent and were consistent with the presence of a trimeric species. Since it is well-known that aryllithium compounds are usually highly aggregated containing electron-deficient carbon-lithium bonds (four-center, two-electron or three-center, two-electron), this information allows us to propose the structure shown in Figure 4 as being the most likely for this compound. Unfortunately, attempted crystallization of $\mathrm{Li}(\mathrm{MeOXL})$ has so far not afforded crystals that were suitable for an X-ray structure determination.

The $100-\mathrm{MHz}^{1} \mathrm{H}$ NMR spectrum of $[\mathrm{Cu}(\mathrm{MeOXL})]_{2}$ and of $\left[\mathrm{Cu}_{2}(\mathrm{MeOXL})_{2} \mathrm{CuBr}\right]_{2}$ in toluene- $d_{8}$ showed at $298 \mathrm{~K}$ only one pattern for the oxazoline moiety for the former and several resonance patterns for the latter (see Table

(24) (a) Jastrzebski, J. T. B. H.; van Koten, G.; Konijn, M.; Stam, C H. J. Am. Chem. Soc. 1982, 104, 5490. (b) Jastrzebski, J. T. B. H.; van Koten, G.; Goubitz, K.; Arlen, C.; Pfeffer, M. J. Organomet. Chem. 1983 $246, \mathrm{C} 75$.

(25) van Koten, G.; Jastrzebski, J. T. B. H.; Noltes, J. G. J. Organomet. Chem. 1977, 140, C23.

(26) Lipshutz, B. H.; Kozlowski, J. A.; Breneman, C. M. J. Am. Chem. Soc. $1985,107,3197$.

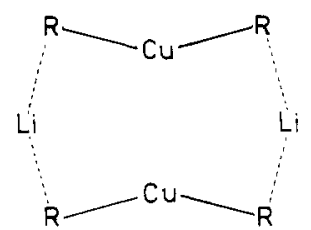

Figure 5. General structures for organocuprates.

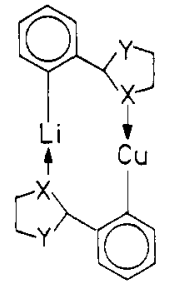

A

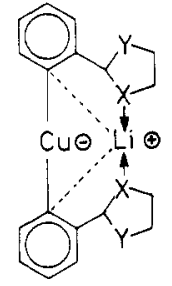

B
Figure 6. Proposed structure for $\left[\mathrm{CuLi}(\mathrm{MeOXL})_{2}\right](4)$.

IV). ${ }^{1} \mathrm{H}$ NMR spectra of these compounds are temperature-dependent which is most likely due to equilibria between aggregated species. Assignment of these patterns was not attempted.

A cryoscopic molecular weight determination in benzene of diethyl ether free cuprate $\left[\mathrm{CuLi}(\mathrm{MeOXL})_{2}\right]$ was consistent with a monomeric species of $\mathrm{CuLi}(\mathrm{MeOXL})_{2}$ stoichiometry. This excludes the possibility that the cuprate has either a dimeric structure as generally proposed for neutral organocuprates (see Figure 5) ${ }^{27}$ and established by $\mathrm{X}$-ray determination for $\mathrm{Cu}_{2} \mathrm{Li}_{2}$ $\left(\mathrm{C}_{6} \mathrm{H}_{4} \mathrm{CH}_{2} \mathrm{NMe}_{2}-2\right)_{4}{ }^{28}$ or, because of its good solubility in benzene, an alternative cation-anion structure, $\left[\mathrm{R}_{2} \mathrm{Cu}\right]^{-}$ $\left[\mathrm{LiL}_{4}\right]^{+29 a}$ Since $[\mathrm{Li}(\mathrm{MeOXL})]_{3}$ and $[\mathrm{Cu}(\mathrm{MeOXL})]_{2}$ were reacted together in a $2: 3$ ratio to form $\left[\mathrm{CuLi}(\mathrm{MeOXL})_{2}\right.$ ], the formation of higher order cuprates of the type $R_{n+m} \mathrm{Cu}_{m} \mathrm{Li}_{n}(n \neq m)$, species which have been found to be present in the ${ }^{1} \mathrm{H}$ NMR spectra of $\mathrm{Me}_{2} \mathrm{CuLi}^{26}$ can be excluded.

The ${ }^{1} \mathrm{H}$ NMR spectrum of [CuLi(MeOXL) $)_{2}$, which contained no signals for $[\mathrm{Li}(\mathrm{MeOXL})]_{3}$ or $[\mathrm{Cu}(\mathrm{MeOXL})]_{2}$, showed a doublet for the MeOXL $\mathrm{CH}_{3}$ protons and an $\mathrm{AB}$ pattern for the $\mathrm{CH}_{2}$ group that points to an inequivalence of the prochiral $\mathrm{CH}_{2}$ protons and also indicates the presence of two equivalent oxazoline groups. Of the two possible structures for this monomeric cuprate shown in Figure 6 structure A can be excluded on the basis of the NMR data. The situation reflected in structure $B$, which we propose for 4 , comprises $\left[\mathrm{R}_{2} \mathrm{Cu}\right]^{-}$and $\left[\mathrm{LiL}_{2}\right]^{+}$units in one molecule. This situation has not been previously identified although it has been reported that $\mathrm{Li}^{+}$can easily be separated from cuprate structures by strongly solvating ligands such as crown ethers ${ }^{29 a}$ and $T H F^{29 b}$ which form $\left[\mathrm{LiL}_{n}\right]^{+}$cations. In the present compound 4 the $\mathrm{Li}$ cation is "solvated" by the heteroatom substituent of the aryl group and is thus kept in proximity of the copper atom. This gives rise to an internal anion-cation separation.

(27) (a) Corey, E. J.; Posner, G. H. J. Am. Chem. Soc. 1968, 90, 5615. (b) Whitesides, G. M.; Fischer, W. F.; Filippo, J. S.; Bashe, R. W.; House H. O. J. Am. Chem. Soc. 1969, 91, 4871. (c) van Koten, G.; Noltes, J. G. J. Chem. Soc., Chem. Commun. 1972, 940. (d) Pearson, R. G.; Gregory, C. D. J. Am. Chem. Soc. 1976, 98, 4098. (e) Stewart, K. R.; Lever, J. R.; Whangbo, M. H. J. Org. Chem. 1982, 47, 1472.

(28) van Koten, G.; Jastrzebski, J. T. B. H.; Muller, F.; Stam, C. H. J. Am. Chem. Soc. 1985, 107, 697 .

(29) (a) Hope, H.; Olmstead, M. M.; Power, P. P.; Sandell, J.; Xu, X. J. Am. Chem. Soc. 1985, 107, 4337. (b) Eaborn, C.; Hitchcock, P. B.; Smith, J. D.; Sullivan, A. D. J. Organomet. Chem. 1984, 263, C23. 
Unfortunately we have not succeeded in obtaining crystals of 4 that were suitable for an X-ray structure determination, and as a consequence we are not sure which site of the oxazoline substituent ( $\mathrm{O}$ or $\mathrm{N}$ ) coordinates to the lithium atom, though it is expected that the harder $\mathrm{O}$ site would bind a $\mathrm{Li}$ cation.

The driving force for the formation of this monomeric cuprate is most likely the more effective bridging than chelating properties of the oxazolinylaryl group and the very effective complexation of the lithium atom by the oxazoline substituent.

In the presence of exactly 1 equivalent of diethyl ether the ${ }^{1} \mathrm{H}$ NMR spectrum of $\left[\mathrm{CuLi}(\mathrm{MeOXL})_{2}\right]$ in toluene- $d_{8}$ at ambient temperature showed together with the pattern for the oxazoline moiety the presence of uncoordinated ether $\left(\delta\left(\mathrm{CH}_{2}\right) 3.26\right.$ and $\delta\left(\mathrm{CH}_{3}\right) 1.11$; compare the values reported for coordinated diethyl ether in $\left[\mathrm{Cu}_{2} \mathrm{Li}_{2}(p-\right.$ $\left.\mathrm{Tol})_{4}\left(\mathrm{Et}_{2} \mathrm{O}\right)_{2}\right] \delta\left(\mathrm{OCH}_{2}\right) 2.72$ and $\left.\delta\left(\mathrm{CH}_{3}\right) 0.64\right){ }^{25}$ Lowering the temperature of this NMR sample containing diethyl ether did not show a shift for the diethyl ether protons, thus excluding the presence of a significant equilibrium between coordinated and free diethyl ether.

Coupling Reactions. To study $\mathrm{C}-\mathrm{C}$ coupling reactions, the reaction of $[\mathrm{Cu}(\mathrm{MeOXL})]_{2}$ with 2-iodopiperonal cyclohexylimine (8b) was selected as a model. Both reactants are expected to contain the representative features of the compounds used by Ziegler et al. $^{5}$ in their studies.

The $1: 1$ reaction of $[\mathrm{Cu}(\mathrm{MeOXL})]_{2}$ with $8 \mathbf{b}$ afforded, after hydrolysis, exclusively the asymmetric coupled product 9. Remarkably, this reaction was completely

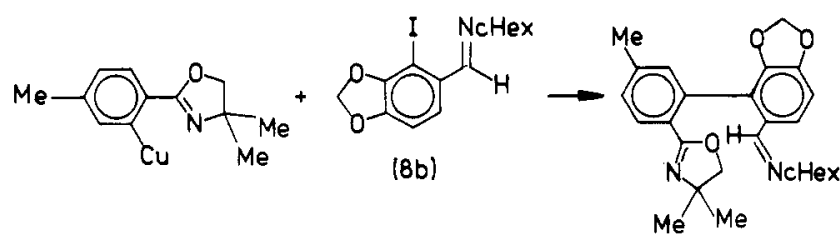

(9) homogeneous, with no observable formation of $\mathrm{CuI}$. Workup of the reaction mixture without hydrolysis afforded a pale yellow compound whose ${ }^{1} \mathrm{H}$ NMR spectrum showed it not to be 9. A considerable dissymmetry in this compound was indicated by the diastereotopism of the $\mathrm{OCH}_{2}$ of the piperonal group and of the $\mathrm{CH}_{2}$ (doublet of doublets) and $\mathrm{CMe}_{2}$ (two singlets) groups of the oxazoline group. Since the same spectrum was also obtained upon the addition of 1 equiv of $\mathrm{CuI}$ to a solution of pure 9 , it is clear that the formation of a $\left(\mathrm{RR}^{\prime}\right) \mathrm{CuI}$ complex occurs concomitantly with the coupling reaction.

When the coupling reaction of $\mathbf{8 b}$ was carried out with $\left[\mathrm{Cu}_{2}(\mathrm{MeOXL})_{2} \mathrm{CuBr}\right]_{2}(3)$, a smooth reaction occurred that quickly resulted in the disappearance of the orange color of 3 and the formation of a white precipitate (most probably $\mathrm{CuBr}$ ), and the biaryl 9 was again formed exclusively.

The coupling reaction of $[\mathrm{Cu}(\mathrm{MeOXL})]_{2}(2 \mathrm{~b})$ and the imine $8 \mathbf{b}$ in the presence of $\mathrm{P}(\mathrm{OMe})_{3}$ also afforded exclusively the $\mathrm{C}-\mathrm{C}$ coupled product 9 , but it appeared that the isolation of well-defined organic or metal-containing products from the reaction of $\mathrm{Li}(\mathrm{MeOXL})$ with $\mathrm{CuBr}\{\mathrm{P}$ $(\mathrm{OMe})_{3}$ \} was not possible. Addition of 1 equiv of $\mathrm{P}(\mathrm{OMe})_{3}$ to an NMR sample of $[\mathrm{Cu}(\mathrm{MeOXL})]_{2}$ resulted in a downfield shift of the MeOXL $\mathrm{CH}_{3}$ and aryl $\mathrm{CH}_{3}$ as well as in broadening of all the signals. These broad signals sharpened upon addition of excess of $\mathrm{P}(\mathrm{OMe})_{3}$ though the $\mathrm{P}(\mathrm{OMe})_{3}$ protons then no longer showed ${ }^{31} \mathrm{P}$ coupling. These observations point to an equilibrium between coordinated and free $\mathrm{P}(\mathrm{OMe})_{3}$ that was investigated further by a microwave titration ${ }^{30}$ of $[\mathrm{Cu}(\mathrm{MeOXL})]_{2}$ with $\mathrm{P}$ -

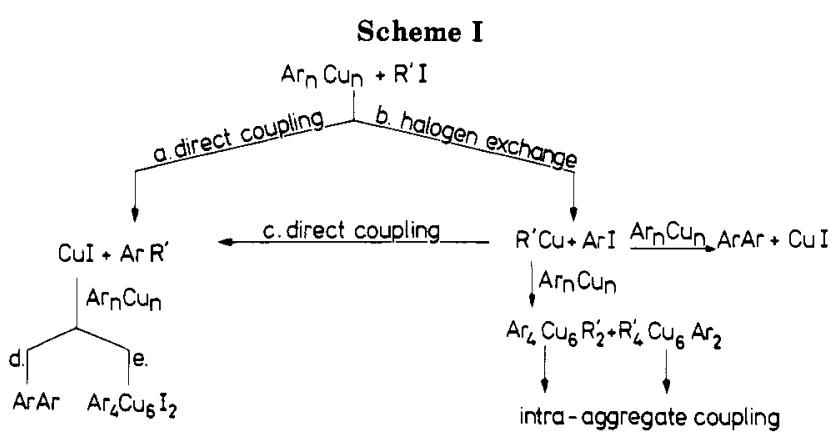

$(\mathrm{OMe})_{3}$. This revealed a 1:1 stoichiometry but does not itself give any information about the actual reaction that has taken place. ${ }^{31}$ Molecular weight determinations of $[\mathrm{Cu}(\mathrm{MeOXL})]_{2}$ with various amounts of $\mathrm{P}(\mathrm{OMe})_{3}$ showed a continuous decrease from a dimeric to a monomeric formulation upon increasing the amount of $\mathrm{P}(\mathrm{OMe})_{3}$ present. It can therefore be concluded from the above results that the addition of $\mathrm{P}(\mathrm{OMe})_{3}$ to $[\mathrm{Cu}(\mathrm{MeOXL})]_{2}$ leads to a breakdown of the organocopper dimer into a monomeric species as follows:

$(\mathrm{MeOXL})_{2} \mathrm{Cu}_{2}+2 \mathrm{P}(\mathrm{OMe})_{3} \rightleftharpoons 2(\mathrm{MeOXL}) \mathrm{Cu}\left[\mathrm{P}(\mathrm{OMe})_{3}\right]$

Since the monomeric species is also in equilibrium with the dimeric organocopper compounds $2 \mathbf{b}$ (see ${ }^{1} \mathrm{H}$ NMR data above), it is not known which of them is the reactive species in the $\mathrm{C}-\mathrm{C}$ coupling reaction. However, considering the very facile coupling observed in the absence of $P$. $(\mathrm{OMe})_{3}$, no critical role of $\mathrm{P}(\mathrm{OMe})_{3}$ in these reactions can be expected.

NMR spectra show no indication that piperonal cyclohexylimine (8a) complexes to the copper dimer [Cu$(\mathrm{MeOXL})]_{2}$. Although this does not exclude the possibility that interaction of the imine substituent with the copper atom preceeds the $\mathrm{C}-\mathrm{C}$ coupling, it does indicate that such an interaction, if present, is not dominant on the NMR time scale. ${ }^{32}$

Regardless of the type of "copper aryloxazoline" used, the reaction with iodopiperonal imines affords the biaryl compound in a very smooth reaction. In view of the very different structural features of $[\mathrm{Cu}(\mathrm{MeOXL})]_{2}$ and $\left[\mathrm{Cu}_{2}-\right.$ $\left.(\mathrm{MeOXL})_{2} \mathrm{CuBr}\right]_{2}$ the success of this reaction under mild conditions therefore cannot be ascribed to the special features provided by the heteroatom substituent. Furthermore, the heteroatom substituent in the aryl iodide does not seem to be important with respect to a possible anchoring function to the copper species.

Proposed routes for the reaction of organocopper compounds $\mathrm{Ar}_{n} \mathrm{Cu}_{n}$ with aryl iodides $\mathrm{R}^{\prime} \mathrm{I}$ are schematically shown in Scheme I. ${ }^{33}$ Previous studies have shown that the first step of this reaction can give either direct coupling (a) or halogen exchange (b). In the latter case ArI would be produced and this could react further with remaining $\mathrm{Ar}_{n} \mathrm{Cu}_{n}$ to afford the symmetric coupled product ArAr. Liberated $\mathrm{CuI}$ can then react with $\mathrm{Ar}_{n} \mathrm{Cu}_{n}$ to afford either the complexed product $\left[(\mathrm{ArCu})_{x}(\mathrm{CuI})_{y}\right]$, which may have a different reactivity to that of $\mathrm{Ar}_{n} \mathrm{Cu}_{n}$, or the $\mathrm{C}-\mathrm{C}$ coupling product ArAr. The important influence of the aryl group

(30) For an example of the use of microwave titrations in the study of organocopper compounds see: van Koten, G.; Jastrzebski, J. T. B. H.; Noltes, J. G. Inorg. Chim. Acta 1977, 21, L9.

(31) Reaction of organocopper compounds with phosphines can give complexation as well as cluster break down: Costa, G.; Camus, A.; Marsich, N.; Gatti, L. J. Organomet. Chem. 1967, 8, 339.

(32) In fact this is also expected since the affinity of the hard $\mathrm{N}$-donor for the soft $\mathrm{Cu}(\mathrm{I})$ will be low.

(33) van Koten, G.; ten Hoedt, R. W. M.; Noltes, J. G. J. Org. Chem. $1977,42,2705$. 
substituents on the occurrence of these various steps has been reported..$^{33}$

In the coupling reactions described by Ziegler et al. no symmetric coupled products were found for arylimines, ${ }^{5}$ which is confirmed by our present study. This implies that the halogen exchange reaction (b) in Scheme I is not of importance since this would lead to reasonable amounts of the symmetric coupled products. Therefore, only the direct coupling path (a) has to be considered. The following reaction of liberated $\mathrm{CuI}$ with $\mathrm{Ar}_{n} \mathrm{Cu}_{n}$ would give rise to the formation of a CuI complexed product, $\mathrm{Ar}_{4} \mathrm{Cu}_{6} \mathrm{I}_{2}$. This latter species has a reactivity comparable to that of $\mathrm{Ar}_{n} \mathrm{Cu}_{n}$, and its formation does not therefore diminish the selectivity and effectiveness of the coupling reaction. These features can explain the high selectivity of the coupling reactions by using copper compounds containing the imine functionality.

Since the ester group compared with the imine group has completely different coordinating properties toward copper(I), the resulting compounds with arylcopper species containing the ester substituent are expected to show different chemical reactivity. For example, in the reaction of aryl ester compounds with arylcopper reagents Ziegler et $a .^{5}$ showed that halogen exchange was significant and this explained the lower selectivity found. Moreover, the lower stability of arylcopper compounds with aryl $\mathrm{O}$ substituents compared to those with aryl $\mathrm{N}$ substituents ${ }^{7}$ may lead to a reasonable contribution of thermal intra-aggregate coupling of the aryl groups.

In conclusion, the heteroatom substituent on an aryl ring essentially influences the stability of its arylcopper species, and it does not behave as an anchoring group in the coupling reaction.

\section{Experimental Section}

Syntheses were carried out by using standard Schlenk techniques in an atmosphere of purified nitrogen. The solvents were dried prior to use. The following compounds were prepared according to literature methods: $\mathrm{CuBr},{ }^{34}$ oxazolines, ${ }^{13}$ and piperonalimines ${ }^{35} n$-BuLi in hexane (1.6 M) was purchased from Janssen Chimica and its concentration checked by the Gilman double-titration method. ${ }^{36}$

${ }^{1} \mathrm{H}$ NMR spectra were obtained on Bruker AC100 and WM250 spectrometers (see Table IV). Molecular weight determinations were performed by cryoscopy in benzene under $\mathrm{N}_{2}$ in a homemade cell, and microwave titrations were carried out on a locally constructed apparatus as described below. Elemental analyses were carried out by the section Elemental Analyses of ITC/TNO, Zeist, The Netherlands.

Lithiation of Oxazolines. The lithiation of the aryloxazolines is exemplified for MeOXL-H affording $\mathrm{Li}(\mathrm{MeOXL})$ (1b). To 4.75 $\mathrm{g}$ of MeOXL-H (25 mmol) dissolved in hexane $(50 \mathrm{ml})$ was added 1 equiv of $n$-BuLi (ca. $1.5 \mathrm{M}$ in hexane) in about $15 \mathrm{~min}$. During the addition a white precipitate was formed. The suspension was stirred for a further $30 \mathrm{~min}$ and subsequently filtered. The precipitate was washed with portions $(20 \mathrm{~mL})$ of hexane until the hexane washings remained colorless. The white solid was dried in vacuo, affording $3.40 \mathrm{~g}$ of the lithium oxazoline compound $1 \mathrm{~b}$, yield $68 \%$.

Molecular weight determination of $\mathrm{Li}(\mathrm{MeOXL}$ ) (cryoscopic in benzene): found, 606 (concentration independent in the range 0.04-0.09 mol $/ \mathrm{L}$ ); calcd, 195 .

Deuteriolysis of the Lithium Oxazolines 1. The lithiated oxazolines $(1 \mathrm{~g}$, about $5 \mathrm{mmol})$ were dissolved in diethyl ether $(30 \mathrm{~mL})$, and an excess of $\mathrm{D}_{2} \mathrm{O}$ was carefully added. The solution was stirred for $15 \mathrm{~min}$ after which the diethyl ether layer was

(34) Brauer, G. Handbuch der Präparatieven Anorganischen Chemie; Ferdinand Enke Verlag: Stuttgart, 1962; Zweiter Auflage, Zweiter Band, p 888 .

(35) Ziegler, F. E.; Fowler, K. W. J. Org. Chem. 1976, 41, 1564.

(36) Müller, E. Methoden Der. Organischen Chemie (Houben-Weyl); Georg Thieme Verlag: Stuttgart, 1970; Band XIII, 1, p 23. separated and dried over $\mathrm{Na}_{2} \mathrm{SO}_{4}$. Removal of the solvent in vacuo afforded the respective deuteriated oxazolines 5 in almost quantitative yield.

Silylation of Lithium Oxazoline $1 \mathrm{~b}$. To a solution of $\mathrm{Li}$ $(\mathrm{MeOXL})(920 \mathrm{mg}, 4.7 \mathrm{mmol})$ in diethyl ether $(40 \mathrm{~mL})$ was added $0.6 \mathrm{~mL}$ of trimethylsilyl chloride $(4.7 \mathrm{mmol})$. The resulting white suspension was refluxed for $4 \mathrm{~h}$ and subsequently filtered. The precipitate $(\mathrm{LiCl})$ was washed with two portions of diethyl ether $(20 \mathrm{~mL})$, and removal of the solvent from the combined filtrates in vacuo afforded the silylated oxazoline 6 as a colorless liquid, yield $1.15 \mathrm{~g}(95 \%)$.

Synthesis of the Copper Oxazolines $2 \mathrm{a}$ and $2 \mathrm{~b}$. To a diethyl ether solution $(150 \mathrm{~mL})$ containing $\mathrm{Li}(\mathrm{MeOXL})(3.50 \mathrm{~g}, 18 \mathrm{mmol})$ was added $\mathrm{CuBr}(2.60 \mathrm{~g}, 18 \mathrm{mmol})$ in ca. $30 \mathrm{~min}$. Initially a white suspension was formed upon addition of $\mathrm{CuBr}$, but this color slowly changed to yellow/orange when about half of the $\mathrm{CuBr}$ had been added. The final reaction mixture was stirred for 2.5 $\mathrm{h}$ and subsequently filtered. The yellow precipitate was washed with one portion of hexane (ca. $50 \mathrm{~mL}$ ) and then dried in vacuo. The yellow compound was redissolved in hot benzene (100-150 $\mathrm{mL}, 75^{\circ} \mathrm{C}$ ). The resulting suspension was filtered hot and the filtrate evaporated to dryness. This afforded $3.92 \mathrm{~g}$ of the light yellow colored organocopper compound 2 , yield $87 \%$. Orange $\mathbf{2 a}$ was obtained by an analogous procedure; yield $70 \%$.

Anal. Calcd for $\mathrm{CuC}_{11} \mathrm{H}_{12} \mathrm{NO}(\mathrm{Cu}(\mathrm{OXL}), 2 \mathrm{a})$ : $\mathrm{C}, 55.56 ; \mathrm{H}, 5.10$; $\mathrm{N}, 5.89 ; \mathrm{O}, 6.73 ; \mathrm{Cu}, 26.73$. Found: C, 54.91; H, 5.24; N, 5.70; O, 6.92; $\mathrm{Cu}, 26.06$. Calcd for $\mathrm{CuC}_{12} \mathrm{H}_{14} \mathrm{NO}$ (Cu(MeOXL), 2b): C, $57.23 ; \mathrm{H}, 5.62 ; \mathrm{N}, 5.56 ; \mathrm{O}, 6.35 ; \mathrm{Cu}, 25.23$. Found: C, 57.44; H, $6.12 ; \mathrm{N}, 5.45 ; \mathrm{O}, 6.42 ; \mathrm{Cu}, 25.08 \%$. Molecular weight determination of $\mathrm{Cu}_{2}(\mathrm{MeOXL})_{2}$ (cryoscopic in benzene): found, 590 (concentration independent in the range 0.04-0.11 mol/L); calcd, 502 .

Synthesis of $\mathrm{CuLi}(\mathrm{MeOXL})_{2}\left(\mathrm{Et}_{2} \mathrm{O}\right)$ (4). (a) From Li(MeOXL) and CuBr. To Li(MeOXL) $(2.4 \mathrm{~g}, 12 \mathrm{mmol})$ in diethyl ether $(125 \mathrm{~mL})$ was slowly added $\mathrm{CuBr}(0.9 \mathrm{~g}, 6 \mathrm{mmol})$. The white suspension was stirred for $1.5 \mathrm{~h}$, and subsequently the precipitate was filtered off. The solid was washed with five portions of diethyl ether $(40 \mathrm{~mL}$ each) and dried in vacuo affording $2.50 \mathrm{~g}$ of an off-white compound, yield $80 \%$. The diethyl ether could be removed by redissolving the compound in benzene and evaporation of the the solution to dryness in vacuo.

Anal. Calcd for $\mathrm{CuLiC}_{28} \mathrm{H}_{38} \mathrm{~N}_{2} \mathrm{O}_{3}: \mathrm{C}, 64.52 ; \mathrm{H}, 7.36 ; \mathrm{N}, 5.37$; $\mathrm{O}, 9.21 ; \mathrm{Cu}, 12.19$. Found: C, $63.65 ; \mathrm{H}, 6.90 ; \mathrm{N}, 5.77 ; \mathrm{O}, 8.57 ; \mathrm{Cu}$, 12.31. Molecular weight determination of diethyl ether free $\mathrm{CuLi}(\mathrm{MeOXL})_{2}$ (cryoscopic in benzene): found, 483 (concentration independent in the range $0.023-0.13 \mathrm{Mol} / \mathrm{L}$ ); calcd, 446 .

(b) From the 1:1 Molar Reaction of $\mathrm{Cu}(\mathrm{OXL})$ with $\mathrm{Li}$ (OXL). To a solution of $\mathrm{Li}(\mathrm{OXL})(1 \mathrm{a})(0.50 \mathrm{~g}, 2.5 \mathrm{mmol})$ in benzene $(30 \mathrm{~mL})$ was added a solution containing $\mathrm{Cu}(\mathrm{OXL})(0.64$ $\mathrm{g}, 2.5 \mathrm{mmol})$ in benzene $(35 \mathrm{~mL})$. The solution was stirred for $1 \mathrm{~h}$ and subsequently evaporated to dryness. The residue was washed with three portions of diethyl ether $(40 \mathrm{~mL}$ each) and dried in vacuo, affording $1.13 \mathrm{~g}$ of yellow compound 4 , yield $85 \%$.

Synthesis of $\left[\mathrm{Cu}_{2}(\mathrm{MeOXL})_{2} \mathrm{CuBr}\right]_{2}(3)$. To a solution of $\mathrm{Cu}(\mathrm{MeOXL})(0.42 \mathrm{~g}, 1.6 \mathrm{mmol})$ in benzene was added $\mathrm{CuBr}(0.12$ $\mathrm{g}, 0.8 \mathrm{mmol}$ ). The light yellow solution turned almost immediately dark red with complete dissolution of the $\mathrm{CuBr}$. Stirring was continued for $1 \mathrm{~h}$ after which traces of metallic copper were removed by filtration and the filtrate was evaporated to dryness in vacuo. Upon addition of hexane to the oily residue, a bright orange solid was formed. This was isolated by filtration and dried in vacuo; yield of $30.50 \mathrm{~g}(90 \%)$.

Anal. Calcd for $\mathrm{Cu}_{3} \mathrm{C}_{24} \mathrm{H}_{28} \mathrm{~N}_{2} \mathrm{O}_{2} \mathrm{Br}$ : C, 44.54; $\mathrm{H}, 4.37 ; \mathrm{N}, 4.33$; $\mathrm{O}, 4.94 ; \mathrm{Br}, 12.34 ; \mathrm{Cu}, 29.46$. Found: C, 44.63; H, 4.54; N, 4.06; $\mathrm{O}, 5.48 ; \mathrm{Br}, 12.86 ; \mathrm{Cu}, 28.95$.

Reaction of $\mathrm{Cu}\left(\mathrm{MeOXL}\right.$ ) (2b) with $\mathrm{Me}_{2} \mathrm{SnBr}_{2}$. To a solution of $\mathrm{Cu}(\mathrm{MeOXL})(0.70 \mathrm{~g}, 2.8 \mathrm{mmol})$ in diethyl ether $(50 \mathrm{~mL})$ was added $\mathrm{Me}_{2} \mathrm{SnBr}_{2}(0.86 \mathrm{~g}, 2.8 \mathrm{mmol})$ dissolved in diethyl ether (20 $\mathrm{mL}$ ). The light yellow suspension was stirred for $15 \mathrm{~min}$ and subsequently evaporated to dryness in vacuo. The remaining residue was extracted with hot benzene $(75 \mathrm{~mL})$. Removal of the benzene from the combined extracts in vacuo and washing of the residue with one portion of hexane $(15 \mathrm{~mL})$ afforded $0.75 \mathrm{~g}$ of a white solid, $7 \mathbf{b}$, yield $65 \%$. The tin compound $7 \mathrm{a}$ was prepared by an analogous procedure from $2 \mathrm{a}$ and $\mathrm{Me}_{2} \mathrm{SnBr}_{2}$.

Procedure for the Coupling Reaction of the Copper Oxazoline Compounds. To a solution of $\mathrm{Cu}(\mathrm{MeOXL})(0.10 \mathrm{~g}, 0.40$ 
mmol) dissolved in THF (20 mL) was added 1 equiv of 2-iodopiperonal cyclohexylimine $(0.14 \mathrm{~g}, 0.40 \mathrm{mmol})$ in one portion. The mixture was stirred overnight and subsequently diluted with $\mathrm{CH}_{2} \mathrm{Cl}_{2}$ (ca. $50 \mathrm{~mL}$ ). This mixture was poured into a $10 \% \mathrm{v} / \mathrm{v}$ solution of $\mathrm{NH}_{4} \mathrm{Cl} / \mathrm{NH}_{4} \mathrm{OH}$. The organic layer was separated and washed with two portions of $\mathrm{NH}_{4} \mathrm{OH} / \mathrm{H}_{2} \mathrm{O}(10 \% \mathrm{v} / \mathrm{v}$ solution $50 \mathrm{~mL}$ ). The organic layer was dried over $\mathrm{Na}_{2} \mathrm{SO}_{4}$ and filtered and the filtrate evaporated to dryness. The crude oily residue was used for recording the ${ }^{1} \mathrm{H}$ NMR spectra. Pure solid coupled product could be obtained by recrystallizing the crude product from $\mathrm{CH}_{2} \mathrm{Cl}_{2}$ /hexane.

${ }^{1} \mathrm{H}$ NMR $\left(\mathrm{C}_{6} \mathrm{D}_{6}\right)$ of pure 9: $\delta 8.29(\mathrm{~s}, 1 \mathrm{H}$, imine $\mathrm{H}), 8.19(\mathrm{~d}$, $\left.1 \mathrm{H}, \mathrm{H}_{6}(\mathrm{OXL}), J=8 \mathrm{~Hz}\right), 8.11\left(\mathrm{~d}, 1 \mathrm{H}, \mathrm{H}_{6}(\mathrm{PI}), J=8 \mathrm{~Hz}\right), 7.10$ $\left(\mathrm{s}, 1 \mathrm{H}, \mathrm{H}_{3}(\mathrm{OXL})\right), 6.88\left(\mathrm{~d}, 1 \mathrm{H}, \mathrm{H}_{5}(\mathrm{OXL}), J=8 \mathrm{~Hz}\right), 6.78(\mathrm{~d}, 1$ $\left.\mathrm{H}, \mathrm{H}_{5}(\mathrm{PI}), J=8 \mathrm{~Hz}\right), 5.32\left(\mathrm{~s}, 2 \mathrm{H}, \mathrm{OCH}_{2} \mathrm{O}\right), 3.54\left(\mathrm{~s}, 2 \mathrm{H}, \mathrm{CH}_{2-}\right.$ (OXL)), 2.90 (br m, $1 \mathrm{H}, \alpha$-H(cHex)), 1.99 (s, $3 \mathrm{H}, \mathrm{CH}_{3}$ (aryl)), 2.0-0.8 (br m, $10 \mathrm{H}, \mathrm{cHex}), 1.07$ and $1.05\left(2 \mathrm{~s}, 6 \mathrm{H}, \mathrm{CH}_{3}(\mathrm{OXL})\right)$.

Synthesis of CuI(RR') Complex. To a solution of $\mathrm{Cu}$ (MeOXL) $(0.13 \mathrm{~g}, 0.5 \mathrm{mmol})$ in benzene $(10 \mathrm{~mL})$ was added 180 $\mathrm{mg}$ of 2-iodopiperonal cyclohexylimine $(0.5 \mathrm{mmol})$. The resulting solution was stirred for $1.5 \mathrm{~h}$ and then evaporated to dryness. The residue was washed with one portion of hexane $(15 \mathrm{~mL})$ affording $0.29 \mathrm{~g}$ of a cream colored solid, yield $95 \%$.

${ }^{1} \mathrm{H}$ NMR $\left(\mathrm{C}_{6} \mathrm{D}_{6}\right): \delta 8.40-8.25\left(\mathrm{br} \mathrm{m}, 3 \mathrm{H}\right.$, imine- $\mathrm{H}, \mathrm{H}_{6}(\mathrm{OXL})$ $\mathrm{H}_{6}$ (PI)), 7.20-6.80 (br m, $3 \mathrm{H}, \mathrm{H}_{3}(\mathrm{OXL}), \mathrm{H}_{5}(\mathrm{OXL}), \mathrm{H}_{5}(\mathrm{PI})$ ), 5.45 and $5.29\left(2 \mathrm{~s}, 2 \mathrm{H}, \mathrm{OCH}_{2} \mathrm{O}\right), 3.57$ and $3.53\left(2 \mathrm{~s}, 2 \mathrm{H}, \mathrm{CH}_{2}(\mathrm{OXL})\right.$ ), $3.35(\mathrm{br} \mathrm{m}, 1 \mathrm{H}, \alpha-\mathrm{H}(\mathrm{cHex})), 1.99\left(\mathrm{~s}, 3 \mathrm{H}, \mathrm{CH}_{3}(\right.$ aryl $), 1.26$ and 1.23 $\left(2 \mathrm{~s}, 6 \mathrm{H}, \mathrm{CH}_{3}(\mathrm{OXL})\right), 2.0-0.9(\mathrm{br} \mathrm{m}, 10 \mathrm{H}, \mathrm{cHex})$.

Description of Microwave Titrations. Cu(OXL) $(0.45 \mathrm{~g}$, $1.9 \mathrm{mmol}$ ) was dissolved in benzene $(60 \mathrm{~mL})$. The solution was brought into the microwave cavity and titrated with a $0.19 \mathrm{M}$ solution of $\mathrm{P}(\mathrm{OMe})_{3}$ in benzene $(65 \mathrm{~mL})$. The titrant was added in portions of $0.25 \mathrm{~mL}$ after which the solution was allowed to stabilize for $25 \mathrm{~s}$. The microwave signal was detected by a crystal detector, and subsequently a new portion of titrant was added. The resulting values were corrected for the change of the polarity of the solution caused by the addition of $\mathrm{P}(\mathrm{OMe})_{3}$ and for the dilution of the solution. The titration of $\mathrm{Cu}(\mathrm{MeOXL})$ was carried out in the same way, using $0.45 \mathrm{~g}$ in $60 \mathrm{~mL}$ of benzene and a 0.29 $M$ solution of $\mathrm{P}(\mathrm{OMe})_{3}$ in benzene $(65 \mathrm{~mL})$.

Crystal Structure Determinations. Crystals of $[\mathrm{Cu}$ $(\mathrm{MeOXL})]_{2}(\mathbf{2 b})$, which were obtained by slow distillation of hexane into a toluene solution containing $2 \mathbf{b}$, are monoclinic, space group $P 2 / n$, with eight formula units $\left(\mathrm{C}_{12} \mathrm{H}_{14} \mathrm{ONCu}\right)$ in a unit cell of dimensions $a=19.050$ (1) $\AA, b=6.671$ (1) $\AA, c=18.927$ (1) $\AA, \beta=106.49(1)^{\circ}, V=2306.4(6) \AA^{3}, D_{\text {calcd }}=1.45 \mathrm{~g} \mathrm{~cm}^{-3}$, and $\mu(\mathrm{Cu} \mathrm{K} \alpha)=23.9 \mathrm{~cm}^{-1}$. A total of 4760 intensities $\left(2.5<\theta<70^{\circ}\right.$; $h,-23$ to $23, k, 0-8, l, 0-23$ ) were measured on a Nonius CAD4 diffractometer employing graphite-monochromatized $\mathrm{Cu} \mathrm{K} \alpha$ radiation. A total of 608 intensities were below the $2.5 \sigma(I)$ level and were treated as unobserved. The dimensions of the crystal were $0.35 \times 0.55 \times 1.0 \mathrm{~mm}$. The structure was solved by using the symbolic addition program SIMPEL, ${ }^{37}$ which yielded the positions of the two Cu atoms and some of the other heavy atoms, and the structure was completed by means of subsequent difference Fourier syntheses. Refinement proceeded through anisotropic block-diagonal least-squares calculations employing unit weights. An empirical absorption correction ${ }^{38}$ was used, and an

(37) Overbeek, A. R.; Schenk, H. In Computing in Crystallography: Schenk, H., Olthoff, R., Koningsveld, H. van, Bassi, G. C., Eds.; Delft University Press: Delft, The Netherlands, 1978.

(38) Walker, N.; Stuart, D. Acta Crystallogr., Sect. A: Found. Crystallogr. 1983, A39, 158. extinction correction was applied. The anomalous dispersion of $\mathrm{Cu}$ was taken into account. The final $R$ value was 0.051 for the 4152 observed reflections. The other program used, apart from SIMPEI, was XRAY76, ${ }^{39}$ and the scattering factors were taken from Cromer and Mann. ${ }^{40}$

Crystals of $\left[\mathrm{Cu}_{2}(\mathrm{MeOXL})_{2} \mathrm{CuBr}\right]_{2}(3)$, which were obtained by slow distillation of hexane into a toluene solution of 3 , were triclinic, space group $P \overline{1}$, with $a=13.661$ (3) $\AA, b=19.154$ (3) $\AA, c=11.665(5) \AA, \alpha=95.46(3)^{\circ}, \beta=92.49(3)^{\circ}, \gamma=110.49(3)^{\circ}$, and $Z=2$. A total of 2103 independent intensities with $I>3 \sigma(I)$ were measured on a Nonius CAD- 4 diffractometer using graphite-monochromatized Mo $\mathrm{K} \alpha$ radiation. The $\mathrm{Cu}$ and $\mathrm{Br}$ positions were derived from a Patterson synthesis, and $C, N$, and $O$ atoms were found from a subsequent $\Delta F$ synthesis. After isotropic block-diagonal least-squares refinements, an empirical absorption correction (DIFABS) ${ }^{38}$ was applied (crystal dimensions $0.3 \times 0.4$ $\times 0.5 \mathrm{~mm} ; \mu=36.5 \mathrm{~cm}^{-1}$ ). Continued anisotropic refinement converged to $R=0.063$. At this stage apart from hydrogen atoms a $\Delta F$ synthesis showed additional electron density in the region $x=-0.048$ to $+0.19, y=0.07-0.22$, and $z=0.11-0.34$. This electron density was presumed to be due to toluene. The electron count was 21.7 , implying about 0.4 molecule of toluene per formula unit. Since the toluene showed disorder and was not of direct interest, no attempts were made to find a satisfactory model for it. Instead, the effect of the solvent was eliminated by correcting the structure factors for its contributions obtained by direct Fourier inversion of its electron density. ${ }^{41}$ Hydrogen atoms were introduced at calculated positions and continued refinement, anisotropic for $\mathrm{Cu}, \mathrm{Br}, \mathrm{N}$, and $\mathrm{O}$ and isotropic for $\mathrm{H}$ converged to $R=0.045\left(R_{\mathrm{w}}=0.078\right)$. The anomalous dispersions of $\mathrm{Cu}$ and $\mathrm{Br}$ were taken into account. An extinction correction was applied, and a weighting scheme $w=1 /\left(13.2+F_{\mathrm{o}}+0.005 F_{\mathrm{o}}{ }^{2}\right)$ was employed.

Acknowledgment. Prof. K. Vrieze and Dr. D. M. Grove are kindly thanked for their stimulating interest in the subject.

Registry No. 1a, 114533-97-0; 1b, 114533-96-9; 2a, 114533-98-1; 2b, 114533-99-2; 3, 106238-68-0; 4a, 114534-01-9; 4b, 114534-00-8; 5a, 56446-23-2; 5b, 114534-04-2; 6, 114534-05-3; 7a (stannane entry), 114534-07-5; 7a (cc entry), 114534-02-0; 7b (stannane entry), 114534-06-4; 7b (cc entry), 114534-03-1; 8b, 58343-44-5; 9, 114534-08-6; OXL-H, 19312-06-2; MeOXL-H, 79568-30-2; CuBr; 7787-70-4; $\mathrm{Me}_{2} \mathrm{SnBr}_{2}, 2767-47-7$.

Supplementary Material Available: Tables of anisotropic thermal parameters for $\mathbf{2 b}$ and $\mathbf{3}$ (Tables $\mathrm{V}$ and VI, respectively), a complete list of interbond distances and angles for $2 b$ (Table IX), a complete list of bond angles for 3 (Table X), a figure of the molecular structure of the second molecule of $\mathbf{2 b}$ (Figure 7), and ORTEP drawings of $\mathbf{2 b}$ and 3 (Figures 8 and 9 , respectively) (13 pages); tables of structure factors for $2 b$ and 3 (Tables VII and VIII, respectively) (37 pages). Ordering information is given on any current masthead page.

(39) Stewart, J. M. The XRAY76 system, Technical Report TRR446; Computing Science Center, University of Maryland, College Park, MD.

(40) Cromer, D. T.; Mann, J. B. Acta Crystallogr., Sect. A: Cryst. Phys., Diffr., Theor. Gen. Crystallogr. 1968, A24, 321 .

(41) The contribution of the solvent density to the scattering is calculated as $F_{h k l}$ olv $=\sum_{i} \sum_{j} \sum_{k} p(i, j, k) \Delta V \cos 2 \pi\left(h \mathrm{x}_{i}+k y_{j}+l z_{k}\right)$. The summation is over all grid points $i, j$, and $k$ within the area of solvent electron density. $(i, j, k)$ is the electron density $\left.(e / \AA)^{3}\right)$ at the grid point $i, j, k . x_{i}, y_{j}$, and $z_{k}$ are the fractional grid point coordinates. $\Delta V$ is the grid volume. 